\title{
The Effect of Seasonal Weather Variation on the Dynamics of the Plague Disease
}

\author{
Rigobert C. Ngeleja, ${ }^{1}$ Livingstone S. Luboobi, ${ }^{1,2}$ and Yaw Nkansah-Gyekye ${ }^{1}$ \\ ${ }^{1}$ Nelson Mandela African Institution of Science and Technology (NM-AIST), Arusha, Tanzania \\ ${ }^{2}$ Department of Mathematics, Makerere University, P.O. Box 7062, Kampala, Uganda \\ Correspondence should be addressed to Rigobert C. Ngeleja; rngeleja@yahoo.com
}

Received 21 February 2017; Accepted 28 June 2017; Published 10 August 2017

Academic Editor: Ram N. Mohapatra

Copyright ( 2017 Rigobert C. Ngeleja et al. This is an open access article distributed under the Creative Commons Attribution License, which permits unrestricted use, distribution, and reproduction in any medium, provided the original work is properly cited.

Plague is a historic disease which is also known to be the most devastating disease that ever occurred in human history, caused by gram-negative bacteria known as Yersinia pestis. The disease is mostly affected by variations of weather conditions as it disturbs the normal behavior of main plague disease transmission agents, namely, human beings, rodents, fleas, and pathogens, in the environment. This in turn changes the way they interact with each other and ultimately leads to a periodic transmission of plague disease. In this paper, we formulate a periodic epidemic model system by incorporating seasonal transmission rate in order to study the effect of seasonal weather variation on the dynamics of plague disease. We compute the basic reproduction number of a proposed model. We then use numerical simulation to illustrate the effect of different weather dependent parameters on the basic reproduction number. We are able to deduce that infection rate, progression rates from primary forms of plague disease to more severe forms of plague disease, and the infectious flea abundance affect, to a large extent, the number of bubonic, septicemic, and pneumonic plague infective agents. We recommend that it is more reasonable to consider these factors that have been shown to have a significant effect on $R_{T}$ for effective control strategies.

\section{Introduction}

Plague is the ancient disease caused by the bacterium Yersinia pestis and has had significant effects on human societies throughout the history [1]. Dynamics of plague disease are the result of complex interactions between human beings, rodent population, flea population, and pathogens in the environment. Seasonal variation particularly temperature, humidity, rainfall, and precipitation greatly affects the normal transmission capacity of plague disease by either lowering it or raising it. It affects pathogen in the environment, fleas, rodents, and even human behavior by altering their normal immigration rate, death rate, survival rate, and infectious capability [2].

1.1. Seasonality in Flea Development Stages and Behavior. Flea's survival is greatly affected by temperature and relative humidity [3]. The ectothermic characteristics of fleas make them very sensitive to temperature fluctuations. Xenopsylla cheopis is the primary vector flea for Yersinia pestis. It is significantly affected by seasonal weather variation as most of its life stages depend on temperature, humidity, and precipitation. The rate of metamorphosis of this kind of flea from egg to adult is also regulated by temperature.

Flea larvae feed on almost any organic debris but mostly they feed on adult excreta which consist of relatively undigested blood [4]. This adult fecal matter when dried falls from the host to serve as food for the larvae. Thus the availability of food (dried flea dirt) for larvae to feed depends on the weather condition particularly temperature and humidity. The larvae develop well in areas where the relative humidity is greater than 75 percent and the temperature is between $21^{\circ} \mathrm{C}$ and $32^{\circ} \mathrm{C}[5,6]$. At constant temperature fleas become most sensitive to air saturation and are massively killed when the air saturation is insufficiency [7]. Considering the fact that all immature flea stages occur outside the host, 
development rates of flea increase with temperature until it reaches a critical value which makes flea most vulnerable. High temperature combined with low humidity hinders flea's survival at immature stages [8].

The condition where relative humidity is below $50 \%$ is unfavorable for flea growth. It is at this condition that the biting rate of flea onto the infected human and rodent or of the infected flea onto the susceptible human and rodent is significantly low. But when the relative humidity is $80 \%$ the flea becomes very active and as a result the biting rate and infection increase significantly. Moreover when temperature is above $27.5^{\circ} \mathrm{C}$ the rapid disappearance of plague bacilli from the flea stomach occurs, resulting in reduced rates of plague disease transmission. This in turn reduces the flea's efficiency in its ability to transmit the plague bacillus to human beings and rodents $[9,10]$.

When fleas are in rodent burrows, their survival of immature stages is affected by soil moisture that is partly controlled by outside precipitation [11]. As a way of getting rid of detrimental moisture losses and temperature swings, rodents normally shift to start living underground [12]. On the other hand, when they are attached with a high organic load, excessively wet conditions in rodent burrows (e.g., relative humidity 95\%) can stimulate the growth of destructive fungi that diminish flea's larval and egg survival [13].

Different studies justify the negative correlation between rainfall and plague epidemics. For example, Cavanaugh and Marshall Jr. [3] reported that, in areas where drains are absent, or where drainage is insufficient as a result of soil composition or impoundments of water, flooding unquestionably causes a drop in the flea population. In areas with improved drainage, such as those with sandy soils, the lessening of the flea population is minimal. Precipitation also influences plague infection for it influences the concentration of rodents, fleas, and humans in the same shelter.

1.2. Seasonality in Rodents. The direct effect posed on rodent population due to temperature change is minor. This is due to the fact that rodents are homoeothermic and hence do not respond immediately to changes in ambient temperatures [14]. Temperature indirectly affects the spread of plague in rodent population in different ways as follows: at a low mean temperature of $10^{\circ} \mathrm{C}$ the bacteria within host (rodent) become very active as a result a large number of infected rodents dying before even the plague bacilli appear in their blood. At this particular temperature rodents also lose the ability to infect other susceptible individuals.

Rainfall may pose positive or negative effect on the increase of rodent population depending on its intensity [11]. A season of moderate rainfall may be considered to affect positively the increase of rodent abundance but when the amount of rainfall is extremely heavy it results in a tremendous rodent population decline [15]. When it is moderate and upon a proper timing, rainfall may foster the increase of rodent population [3]. This is due to the fact that rodent's reproduction period normally follows wet seasons [16-18]. That is to say, the increase of rodent population during wet period is expected to be higher than that during the dry seasons. This clearly concurs with the result in the study by Leirs et al. [19], which narrates that, in Tanzania, rodent population densities show clear association with the annual rainfall and its seasonal distribution. However when rainfall is of high intensity, it causes flooding of rodent burrows. Large number of rodents population dies and the remaining ones normally move from forest to the households where they can protect themselves $[3,8,20]$. In other cases, increased precipitation or drought stalwartly disturbs rodent population dynamics, as it deters food availability.

1.3. Seasonality in Pathogens in the Environment. When the bacteria are in lungs, the transmission of Yersinia pestis is possible through various ways: contact transmission, in which one may be infected through physical contact with respiratory particles on the infected surface; airborne transmission, which occurs through inhaling the bacteria causing the disease through successive contact with the nose or mouth of an infected individual; respiratory particles, which occurs through respiratory droplets which is through shedding of respiratory particles (i.e., droplets or aerosols) from an infected human or rodent into the environment [21].

Extreme temperatures regularly are ruinous to the survival of pathogens causing plague. The changes in temperature may lead to varying effects on the pathogens in the environment and vectors that live in an environment. When the mean temperature approaches the maximum limit that can be endured by the pathogens, a small increase in temperature may be very dangerous to the pathogen survival. Conversely when pathogens are in the environment characterized by low mean temperature, a small increase in temperature may result in increased development, incubation, and replication of the pathogen in the environment $[22,23]$.

Davis [24] compared the seasonal incidence of plague with usual atmospheric conditions in particular temperature and rainfall. It was depicted that human plague is more frequent in warm moist weather between $15^{\circ} \mathrm{C}$ and $27^{\circ} \mathrm{C}$ than in hot dry (over $27^{\circ} \mathrm{C}$ ) or cold weather (under $15^{\circ} \mathrm{C}$ ). Mitscherlich and Marth [25] narrate that the solar exerts a detrimental effect on bacterial aerosol and the decay rate of Yersinia pestis is proportional to the increase of UV light.

The reports by Ayyadurai et al. [26] and Mollaret [27] justify the ability of the Yersinia pestis to culture the organism from deep within contaminated soil. Eisen et al. [28] were able to show the great potential durability of Yersinia pestis in the soil substrate. The long duration of their survival in the soil supports indirectly the virulence maintenance.

Yersinia pestis exhibit a very slow growth at the temperature between $35^{\circ} \mathrm{C}$ and $37^{\circ} \mathrm{C}$ but they grow very fast at the temperature $28^{\circ} \mathrm{C}$. They die very rapid if exposed to a UV light or temperature exceeding $40^{\circ} \mathrm{C}$ or when exposed to intensive desiccation [29-31]. Bacteria decrease their sensitivity when the level of humidity drops below 76\% [25].

When an infected individual coughs or sneezes, thousands of the bacteria are released in air [32]. The released respiratory particles may be large and heavy that they cannot 
remain suspended in the air. When respiratory particles are large the transmission can only occur when these particles are expelled directly onto another close susceptible individual. In some cases the release of smaller respiratory particles may occur; this is when the airborne transmission is possible. The smaller released particles are easily suspended in the air respired (i.e., passed to the lower respiratory tract) [33].

Relative humidity and temperature affect the transmission of Yersinia pestis from one individual to the other. Humidity affects the size of the respiratory particle [34]. When humidity is low the large drops partially evaporate to create smaller, lighter drops that are more likely to remain airborne for extended periods of time [35]. That is to say, when the air is sufficiently dry the large sized particles shrink to a size that favors long-range transport which in turn leads to increased infection.

1.4. Seasonality in Human Behavior. Human activities and behavior in plague-infected areas are also to be considered as important determinants of plague transmission to and by humans [42]. When occurrences of plague are due to human intrusions in natural plague areas, it is thus important to consider season variation as a second-order variable that influences disease incidence through human behavior. In Tanzania drought and famine which are the result of lack of rainfall and temperature fluctuation have a great impact on the farmers and pastoralists as they force them to move from one area to another searching for food for themselves and their cattle. These human intrusions from one place to another may lead to the increase of plague disease transmission in rodents, fleas, human population, and pathogens in the environment.

\section{Model Formulation}

We describe the complex interaction that leads to plague disease transmission and use it to formulate a model for the dynamics of the plague disease coupled with the effect of seasonal weather variation in its transmission. The model includes four populations, namely, human beings, rodents, fleas, and pathogens, in the environment. We generally assume that all individuals from each population are susceptible to the disease, the recovered individuals confer temporary immunity and return to be susceptible again, and the infectious are all individuals with either bubonic plague or pneumonic or septicemic plague.

2.1. Variables and Parameters Used in the Model. In Notations and Table 1 we present variables and parameters, their description, and their values as used in the model. We have obtained the parameter values from the literature that relate to this study and the present information on plague disease and through estimation.

2.2. Model Description. The human population is divided into six subgroups: the subgroup of people who have not contracted the disease, to be referred to as susceptible and denoted by $S_{H}$, but may get it if they come into contact with $I_{H S}, I_{H P}, I_{R S}, I_{H P}, I_{F}$, or $A$; people who have the disease but have not shown any symptom and are incapable of transmitting the disease to be referred to as exposed and denoted by $E_{H}$; those who are infected and capable of transmitting the disease are divided into three subgroups: there are those who have bubonic plague denoted by $I_{H B}$, those with septicemic plague denoted by $I_{H S}$, and those who have pneumonic plague disease denoted by $I_{H P}$. The fraction of population in $I_{H B}$ if treated or through strong body immunity may recover and move to subgroup $R_{H}$; otherwise they progress either to a septicemic disease infective agent $I_{H S}$ or to pneumonic plague disease infective agent $I_{H P}$ or else they die. The population in the subgroup $I_{H S}$ through strong body immunity or if treated recover and progress to the subgroup $R_{H}$ and if not treated they progress and join subgroup $I_{H P}$; otherwise they die. The population of the subgroup $I_{H P}$ is considered as a very dangerous stage of plague disease; it is a very fatal stage of plague disease with the fatality rate of about $100 \%$; however if treated they recover and join subgroup $R_{H}$; otherwise they die. So the total human population $N_{1}$ is as given by

$$
N_{1}=S_{H}+E_{H}+I_{H B}+I_{H S}+I_{H P}+R_{H} \text {. }
$$

Fleas are divided into two subgroups, those who have not contracted the disease but may get it if they get in contact with infectious agent (rodent or human) referred to as susceptible flea and denoted by $S_{F}$ and those who are infected and are capable of transmitting the disease referred to as infective agents and denoted by $I_{F}$. The total flea population $N_{2}$ is as given by

$$
N_{2}=S_{F}+I_{F}
$$

The rodents are divided into five subgroups; those who have not contracted the disease but may get it if they get in contact with $I_{H S}, I_{H P}, I_{R S}, I_{H P}, I_{F}$, or $A$, referred to as susceptible rodents and denoted by $S_{R}$; those who have the disease but have not shown any symptom and are incapable of transmitting the disease referred to as exposed and denoted by $E_{R}$; those who are infected and capable of transmitting the disease are divided into three subgroups: those who have bubonic plague denoted by $I_{R B}$, those with septicemic plague denoted by $I_{R S}$, and those who have pneumonic plague $I_{R P}$. The fraction of population in $I_{R B}$ may progress either to a septicemic plague disease infective agent $I_{R S}$ or to pneumonic plague disease infective agent $I_{R P}$. The rodent population in the subgroup $I_{R S}$ may either progress to pneumonic plague disease infective agent $I_{R P}$; otherwise they die. The population in the subgroup $I_{R P}$ is considered as a very dangerous stage of plague disease and very fatal so the mortality due to disease in this subgroup is approximated to be $100 \%$. Then the total rodent population $N_{3}$ is as given by

$$
N_{3}=S_{R}+E_{R}+I_{R B}+I_{R S}+I_{R P}
$$

The individuals with pneumonic plague may release pathogens causing plague disease to the environment denoted by $A$ through coughing or sneezing. When the condition in soil/environment is favorable, pathogens may 
TABle 1: Parameters and their description.

\begin{tabular}{|c|c|c|c|}
\hline Parameters & Description & Value & Reference/source \\
\hline$\Gamma_{r b f}(t)$ & Adequate contact rate: between $I_{R B}$ and flea & 0.1 & Eisen et al. [36] \\
\hline$\Gamma_{r s f}(t)$ & Adequate contact rate: between $I_{R S}$ and flea & 0.1 & Eisen et al. [36] \\
\hline$\Gamma_{f h}(t)$ & Adequate contact rate: between $I_{F}$ and human & 0.0641 & Eisen et al. [36] \\
\hline$\Gamma_{f r}(t)$ & Adequate contact rate: between $I_{F}$ and rodent & 0.0641 & Eisen et al. [36] \\
\hline$\Gamma_{h p h}(t)$ & Adequate contact rate: between $I_{H P}$ and $S_{H}$ & 0.39 & Estimated \\
\hline$\Gamma_{h s h}(t)$ & Adequate contact rate: between $I_{H S}$ and $S_{H}$ & 0.12 & Estimated \\
\hline$\Gamma_{r b h}(t)$ & Adequate contact rate: between $I_{R B}$ and $S_{H}$ & & \\
\hline$\Gamma_{r p h}(t)$ & Adequate contact rate: between $I_{R P}$ and $S_{H}$ & 0.19 & Estimated \\
\hline$\Gamma_{r s h}(t)$ & Adequate contact rate: between $I_{R S}$ and $S_{H}$ & 0.21 & Estimated \\
\hline$\alpha_{1}$ & Progression rate of $S_{H}$ to $E_{H}$ population & 0.99 & Estimated \\
\hline$\alpha_{2}$ & Progression rate out of $E_{H}$ to infectious state & 0.23 & Gani and Leach [37] \\
\hline$\rho_{1} \alpha_{3}$ & Progression rate out of $I_{H B}$ to $I_{H P}$ & & \\
\hline$\rho_{2} \alpha_{3}$ & Progression rate out of $I_{H B}$ to $R_{H}$ & & \\
\hline$\rho_{3} \alpha_{3}$ & Progression rate out of $I_{H B}$ to $I_{H S}$ & & \\
\hline$\delta_{1 b}$ & Disease induced death rate of $I_{H B}$ & 0.04 & Keeling and Gilligan [38] \\
\hline$\alpha_{4}$ & Progression rate out of $I_{H S}$ to $I_{H P}$ and $R_{H}$ & 0.06 & Estimated \\
\hline$\delta_{1 s}$ & Disease induced death rate of $I_{H S}$ & 0.04 & Estimated \\
\hline$\alpha_{5}$ & Progression rate out of $I_{H P}$ to $R_{H}$ & 0.4 & Gani and Leach [37] \\
\hline$\delta_{1 p}$ & Disease induced death rate of $I_{H P}$ & 0.63 & Kugeler et al. [39] \\
\hline$\gamma_{1}$ & Progression rate of $S_{R}$ to $E_{R}$ & 0.92 & Estimated \\
\hline$\Gamma_{h b f}(t)$ & Adequate contact rate: between $I_{H B}$ and flea & 0.1 & Eisen et al. [36] \\
\hline$\Gamma_{h s f}(t)$ & Adequate contact rate: between $I_{H S}$ and flea & 0.1 & Eisen et al. [36] \\
\hline$\Gamma_{r p r}(t)$ & Adequate contact rate: between $I_{R P}$ and $S_{R}$ & 0.9 & Estimated \\
\hline$\Gamma_{r s r}(t)$ & Adequate contact rate: between $I_{R S}$ and $S_{R}$ & 0.9 & Estimated \\
\hline$\Gamma_{h p r}(t)$ & Adequate contact rate: between $I_{H P}$ and $S_{R}$ & 0.00005 & Estimated \\
\hline$\Gamma_{h s r}(t)$ & Adequate contact rate: between $I_{H S}$ and $S_{R}$ & 0.00008 & Estimated \\
\hline$\gamma_{2}$ & The rate at which rodent becomes infectious & 0.98 & Estimated \\
\hline$\gamma_{3}$ & Progression rate out of $I_{R B}$ to $I_{R S}$ and $I_{R P}$ & 0.194 & Tollenaere et al. [40] \\
\hline$\delta_{3 b}$ & Disease induced death rate of $I_{R B}$ & 0.1 & Estimated \\
\hline$\gamma_{4}$ & Progression rate out of $I_{R S}$ to $I_{R P}$ & 0.05 & Estimated \\
\hline$\delta_{3 s}$ & Disease induced death rate of $I_{R S}$ & 73 & Tollenaere et al. [40] \\
\hline$\delta_{3 p}$ & Disease induced death rate of $I_{R P}$ & 0.14 & Estimated \\
\hline$\omega$ & Progression rate of $R_{H}$ to $S_{H}$ & 0.33 & Kugeler et al. [39] \\
\hline$\mu_{1}$ & Natural death rate for human being & 0.04 & Keeling and Gilligan [38] \\
\hline$\mu_{2}$ & Natural death rate for flea & 0.2 & Bacot and Martin [7] \\
\hline$\mu_{3}$ & Natural death rate for rodent & 1 & Morand and Harvey [41] \\
\hline$\omega_{1}(t)$ & Adequate contact rate: $A$ and human being & & \\
\hline$\omega_{2}(t)$ & Adequate contact rate: $A$ and rodent & & \\
\hline$\eta_{1}(t)$ & Recruitment rate of $A$ by $I_{H P}$ & 0.2 & Estimated \\
\hline$\eta_{2}(t)$ & Recruitment rate of $A$ by $I_{R P}$ & 0.4 & Estimated \\
\hline$\mu_{4}$ & Natural death rate for pathogens & 0.1 & Estimated \\
\hline$\psi_{1}$ & Recruitment rate of human beings & 0.09 & Estimated \\
\hline$\psi$ & Recruitment rate of fleas & & \\
\hline$\psi_{3}$ & Recruitment rate of rodents & & \\
\hline
\end{tabular}


remain infectious in the environment for a long time. When a susceptible individual adequately interacts with the environment infested with Yersinia pestis, he/she gets the disease even in the absence of any vector.

2.3. Description of Interactions. The susceptible fleas in subgroup $S_{F}$ get Yersinia pestis bacteria through biting the infected rodent $I_{R B}$ or $I_{R S}$ who are the primary reservoir for the bacteria and become infected at the rates $\Gamma_{r b f}$ and $\Gamma_{r s f}$, respectively. Fleas may also get the disease when they bite the infected human being with bubonic plague $I_{H B}$ or septicemic plague $I_{H S}$ at the rates $\Gamma_{h b f}$ and $\Gamma_{h s f}$, respectively. Thus the flea population gets plague infection with the force of infection given in

$$
\begin{aligned}
G_{3}(t)= & \frac{\Gamma_{h b f}(t) I_{H B}+\Gamma_{h s f}(t) I_{H S}}{N_{1}} \\
& +\frac{\Gamma_{r b f}(t) I_{R B}+\Gamma_{r s f}(t) I_{R S}}{N_{3}} .
\end{aligned}
$$

The human population may get the disease in one of the following ways: when the infected flea $I_{F}$ bites and infects the susceptible human being $S_{H}$ at a rate $\Gamma_{f h}$; when they interact with one another; this can be with either a person with pneumonic plague $I_{H P}$ through airborne transmission or septicemic plague $I_{H S}$ through physical or sexual contact at the rates $\Gamma_{h p h}$ and $\Gamma_{h s h}$, respectively. Another infection is through airborne transmission through interaction with rodent infected with pneumonic plague $I_{R P}$ or through touching or eating the infected rodent with septicemic plague $I_{R S}$ at rates of $\Gamma_{r p h}$ and $\Gamma_{r s h}$, respectively. Human beings may also get the infection from the environment when they breath in the bacteria or physically contact the infected material at the rate of $\omega_{1}$. That is to say, human population acquire plague disease following effective contact with infected human, rodent, flea, and the environment with force of infection $G_{1}$ given by

$$
\begin{aligned}
G_{1}(t)= & \frac{\Gamma_{h p h}(t) I_{H P}+\Gamma_{h s h}(t) I_{H S}}{N_{1}}+\Gamma_{f h}(t) \frac{I_{F}}{N_{2}} \\
& +\frac{\Gamma_{r p h}(t) I_{R P}+\Gamma_{r s h}(t) I_{R S}}{N_{3}}+\omega_{1}(t) A .
\end{aligned}
$$

The subgroup $S_{H}$, after the infection, progresses and becomes latent to the disease at a rate $\alpha_{1}$. After 2 to 7 days the subgroups $E_{H}$ become infected into one of the three infectious classes, $I_{H B}, I_{H S}$, or $I_{H P}$ (depending on the mode of transmission an individual is exposed to), and are capable of transmitting the disease. The proportion of $E_{H}$ progresses and becomes infected by bubonic plague $I_{H B}$, septicemic plague $I_{H S}$, or pneumonic plague $I_{H P}$ at the rate $\alpha_{2}$ and proportion to $v_{1}, v_{2}$, or $v_{3}$, respectively. The compartment $I_{H B}$ either through strong body immunity or if they get treatment they recover and move to subgroup $R_{H}$ at a rate $\alpha_{3}$; otherwise they either progress to subgroup $I_{H P}$ or $I_{H S}$ at a rate $\alpha_{3}$ or die either naturally at a rate $\mu_{1}$ or due to the disease at a rate $\delta_{1 b}$. The fraction of humans with septicemic plague $I_{H S}$ either through strong body immunity or if treated recover at a rate $\alpha_{4}$ and join $R_{H}$; otherwise they either progress to subgroup $I_{H P}$ at a rate $\alpha_{4}$ or die due to the disease at a rate $\delta_{1 s}$ or naturally at a rate $\mu_{1}$. The compartments $I_{H P}$ if treated recover at a rate $\alpha_{5}$; otherwise they die either naturally at a rate $\mu_{1}$ or due to the disease at a rate $\delta_{1 p}$. The subgroup $R_{H}$ attain temporary immunity and then return and become susceptible $S_{H}$ at a rate $\omega$.

The rodent population may get a disease in one of the following ways: when the infected flea $I_{F}$ bites and infects the susceptible rodent $S_{R}$ at a rate $\Gamma_{f r}$, through interaction between rodents themselves, which may be with rodent infected by pneumonic plague $I_{R P}$ or septicemic plague $I_{R S}$ at the rates $\Gamma_{r p r}$ and $\Gamma_{r s r}$, respectively. The other infection may be through interaction with human infected with either pneumonic plague $I_{H P}$ or septicemic plague $I_{H S}$ at rates of $\Gamma_{h p r}$ and $\Gamma_{h s r}$, respectively. When the susceptible rodent sufficiently interacts with the pathogens in environment through breathing in the bacteria or physically touches the infected material, it gets the infections at the rate of $\omega_{2}$. Rodent also gets the disease through adequate interaction with rodent, human, flea, and pathogens in the environment with force of infection $G_{2}$ given by

$$
\begin{aligned}
G_{2}(t)= & \frac{\Gamma_{h p r}(t) I_{H P}+\Gamma_{h s r}(t) I_{H S}}{N_{1}}+\Gamma_{f r}(t) \frac{I_{F}}{N_{2}} \\
& +\frac{\Gamma_{r p r}(t) I_{R P}+\Gamma_{r s r}(t) I_{R S}}{N_{3}}+\omega_{2}(t) A .
\end{aligned}
$$

The subgroup $S_{R}$, after the infection, progress and become latent to the disease at a rate $\gamma_{1}$. After 2 to 7 days the subgroup $E_{R}$ become infected and capable of transmitting the disease; the fraction of it progresses and becomes infected by bubonic plague $I_{R B}$, septicemic plague $I_{R S}$, or pneumonic plague $I_{R P}$ at the rate $\gamma_{2}$ and proportional to $\tau_{1}, \tau_{2}$, or $\tau_{3}$ respectively. The rodent in subgroup $I_{R B}$ may either progress to subgroup $I_{R P}$ or $I_{R S}$ at a rate $\gamma_{3}$ or die either naturally at a rate $\mu_{3}$ or due to the disease at a rate $\delta_{3 b}$. The compartment $I_{R S}$ may either progress to $I_{R P}$ at a rate $\gamma_{4}$ or die due to a disease at a rate $\delta_{3 s}$ or naturally at a rate $\mu_{3}$ and the compartments $I_{R P}$ die either naturally at a rate $\mu_{3}$ or due to the disease at a rate $\delta_{3 p}$.

With regard to the pathogens in the environment, we assume that the adequate interaction with $S_{H}$ and $S_{R}$ has a negligible effect on the dynamics of pathogens population size in the environment. The pathogens in the environment are populated at a constant rate $\lambda_{4}$. The infected human with pneumonic plague $I_{H P}$ and rodent with pneumonic plague $I_{R P}$ also populate the environment $A$ with the bacteria at the rates $\eta_{1}$ and $\eta_{2}$, respectively. Thus the environment is populated with pathogens causing plague disease with the force of infection $G_{4}$ given by

$$
G_{4}(t)=\lambda_{4}(t)+\eta_{1}(t) \frac{I_{H P}}{N_{1}}+\eta_{2}(t) \frac{I_{R P}}{N_{3}} .
$$

The pathogens within the environment suffer natural mortality at a rate $\mu_{4}$. Human population in subgroups $S_{H}$ and $E_{H}$, flea population in subgroup $S_{F}$, and rodent population in subgroups $S_{R}$ and $E_{R}$ suffer natural mortality at rates $\mu_{1}, \mu_{2}$, 
and $\mu_{3}$, respectively. The compartments $I_{H B}, I_{H S}, I_{H P}, I_{F}, I_{R B}$, $I_{R S}$, and $I_{R P}$ suffer both natural death at the rates $\mu_{1}, \mu_{2}$, and $\mu_{3}$ and disease induced mortality at rates $\delta_{1 b}, \delta_{1 s}, \delta_{1 p}, \delta_{2}, \delta_{3 b}, \delta_{3 s}$, and $\delta_{3 p}$ respectively. Human, flea, and rodent are recruited at the rates $\psi_{1}, \psi_{2}$, and $\psi_{3}$, respectively.

2.4. Model Equations for Plague Disease. Now we assume that the variation of infection capability from one individual to the other, migration of individuals from one place to another, and recruitment and death rates of individuals in different stages due to seasonal weather variation affect only the rate at which the disease is transmitted from one infected individual to the other. We now use the variables and parameters and their description given in Notations and Table 1 and the description of interactions to drive the system of differential equations given as follows.

\section{Human Beings}

$$
\begin{aligned}
\frac{d S_{H}}{d t}= & \sigma_{1} \psi_{1}+\omega R_{H}-\alpha_{1} G_{1}(t) S_{H}-\mu_{1} S_{H}, \\
\frac{d E_{H}}{d t}= & \left(1-\sigma_{1}\right) \psi_{1}+\alpha_{1} G_{1}(t) S_{H}-\alpha_{2} E_{H}-\mu_{1} E_{H}, \\
\frac{d I_{H B}}{d t}= & \alpha_{2} v_{2} E_{H}-\alpha_{3} I_{H B}-\left(\mu_{1}+\delta_{1 b}\right) I_{H B}, \\
\frac{d I_{H S}}{d t}= & \alpha_{3} \rho_{3} I_{H B}+\alpha_{2} \nu_{3} E_{H}-\alpha_{4} I_{H S}-\left(\mu_{1}+\delta_{1 s}\right) I_{H S}, \\
\frac{d I_{H P}}{d t}= & \alpha_{2} v_{1} E_{H}+\alpha_{3} \rho_{1} I_{H B}+\alpha_{4} \xi I_{H S}-\alpha_{5} I_{H P} \\
& -\left(\mu_{1}+\delta_{1 p}\right) I_{H P}, \\
\frac{d R_{H}}{d t}= & \alpha_{3} \rho_{2} I_{H B}+\alpha_{4}(1-\xi) I_{H S}+\alpha_{5} I_{H P}-\omega R_{H} \\
& -\mu_{1} R_{H} .
\end{aligned}
$$

Rodents

$$
\begin{aligned}
\frac{d S_{R}}{d t}= & \sigma_{2} \psi_{3}-\gamma_{1} G_{2}(t) S_{R}-\mu_{3} S_{R} \\
\frac{d E_{R}}{d t}= & \left(1-\sigma_{2}\right) \psi_{3}+\gamma_{1} G_{2}(t) S_{R}-\gamma_{2} E_{R}-\mu_{3} E_{R} \\
\frac{d I_{R B}}{d t}= & \gamma_{2} \tau_{3} E_{R}-\gamma_{3} I_{R B}-\left(\mu_{3}+\delta_{3 b}\right) I_{R B} \\
\frac{d I_{R S}}{d t}= & \gamma_{2} \tau_{2} E_{R}+\gamma_{3}(1-\phi) I_{R B}-\gamma_{4} I_{R S} \\
& -\left(\mu_{3}+\delta_{3 s}\right) I_{R S}
\end{aligned}
$$

$$
\frac{d I_{R P}}{d t}=\gamma_{2} \tau_{1} E_{R}+\gamma_{3} \phi I_{R B}+\gamma_{4} I_{R S}-\left(\mu_{3}+\delta_{3 p}\right) I_{R P}
$$

Fleas

$$
\begin{aligned}
& \frac{d S_{F}}{d t}=\psi_{2 s}-\beta G_{3}(t) S_{F}-\mu_{2} S_{F}, \\
& \frac{d I_{F}}{d t}=\psi_{2 i}+\beta G_{3}(t) S_{F}-\left(\mu_{2}+\delta_{2}\right) I_{F} .
\end{aligned}
$$

Pathogens

$$
\frac{d A}{d t}=\lambda_{4}(t)+\frac{\eta_{1}(t) I_{H P}}{N_{1}}+\frac{\eta_{3}(t) I_{R P}}{N_{3}}-\mu_{4}(t) A
$$

\section{Basic Properties of the Model}

In this section we discuss the feasible region and positivity of the plague disease model. For convenience purpose and easy presentation of the result we let $C$ denote all continuous functions on the real line. If $f$ is a periodic function in $C$ then we use $\bar{f}$ for the average value of $f$ on time interval $[0, T]$ defined by

$$
\bar{f}=\frac{1}{T} \int_{0}^{T} f(t) d t
$$

for a continuous $T$-periodic function $f(t)$.

3.1. Invariant Region. Plague disease affects human, rodent, flea, and pathogens in the environment populations. For the possible modeling process all state variables and parameters of the model must be nonnegative for $\forall t \geq 0$. We thus need to verify whether the solutions of the model system ((8a), (8b), (8c), (8d), (8e), (8f))-(11) are in suitable feasible region where all state variables are positive. Inspired by Dumont et al. [43] and Mpeshe et al. [44] we first write system ((8a), (8b), (8c), (8d), (8e), (8f))-(11) in the following compact form:

$$
\frac{d X}{d t}=A(x) X+F
$$

where $X=\left(S_{H}, E_{H}, I_{H B}, I_{H S}, I_{H P}, R_{H}, S_{R}, E_{R}, I_{R B}, I_{R S}, I_{R P}\right.$, $\left.S_{F}, I_{F}, A\right)^{T}, A(x)$ is a $14 \times 14$ matrix, and $F$ is a column vector. We then have

$$
A(x)=\left(\begin{array}{ll}
A_{11} & A_{12} \\
A_{21} & A_{22}
\end{array}\right)
$$


where

$$
\begin{aligned}
& \mathbf{A}_{11}=\left(\begin{array}{ccccccc}
-g_{1} & 0 & 0 & 0 & 0 & \omega & 0 \\
\alpha_{1} G_{1}(t) & -\left(\alpha_{2}+\mu_{1}\right) & 0 & 0 & 0 & 0 & 0 \\
0 & \alpha_{2} \nu_{2} & -a_{1} & 0 & 0 & 0 & 0 \\
0 & \alpha_{2} \nu_{3} & \rho_{3} \alpha_{3} & -a_{2} & 0 & 0 & 0 \\
0 & \alpha_{2} \nu_{1} & \rho_{1} \alpha_{3} & \alpha_{4} \xi & -a_{3} & 0 & 0 \\
0 & 0 & \rho_{2} \alpha_{3} & \alpha_{4}(1-\xi) & \alpha_{5} & -\left(\omega+\mu_{1}\right) & 0 \\
0 & 0 & 0 & 0 & 0 & 0 & -g_{2}
\end{array}\right) \\
& \mathbf{A}_{12}=\left(\begin{array}{ccccccc}
0 & 0 & 0 & 0 & 0 & 0 & 0 \\
0 & 0 & 0 & 0 & 0 & 0 & 0 \\
0 & 0 & 0 & 0 & 0 & 0 & 0 \\
0 & 0 & 0 & 0 & 0 & 0 & 0 \\
0 & 0 & 0 & 0 & 0 & 0 & 0 \\
0 & 0 & 0 & 0 & 0 & 0 & 0 \\
0 & 0 & 0 & 0 & 0 & 0 & 0
\end{array}\right) \\
& \mathbf{A}_{22}=\left(\begin{array}{ccccccc}
-\left(\gamma_{2}+\mu_{3}\right) & 0 & 0 & 0 & 0 & 0 & 0 \\
\gamma_{2} \tau_{3} & -a_{4} & 0 & 0 & 0 & 0 & 0 \\
\gamma_{2} \tau_{2} & \gamma_{3}(1-\phi) & -a_{5} & 0 & 0 & 0 & 0 \\
\gamma_{2} \tau_{1} & \gamma_{3} \phi & \gamma_{4} & -\left(\mu_{3}+\delta_{3 p}\right) & 0 & 0 & 0 \\
0 & 0 & 0 & 0 & -g_{3} & 0 & 0 \\
0 & 0 & 0 & 0 & \beta G_{3}(t) & -\left(\mu_{2}+\delta_{2}\right) & 0 \\
0 & 0 & 0 & \frac{\eta_{2}(t)}{N_{3}} & 0 & 0 & -\mu_{4}
\end{array}\right), \\
& \mathbf{A}_{21}=\left(\begin{array}{ccccccc}
0 & 0 & 0 & 0 & 0 & 0 & \gamma_{1} G_{2}(t) \\
0 & 0 & 0 & 0 & 0 & 0 & 0 \\
0 & 0 & 0 & 0 & 0 & 0 & 0 \\
0 & 0 & 0 & 0 & 0 & 0 & 0 \\
0 & 0 & 0 & 0 & 0 & 0 & 0 \\
0 & 0 & 0 & 0 & 0 & 0 & 0 \\
0 & 0 & \frac{\eta_{1}(t)}{N_{1}} & 0 & 0 & 0 & 0
\end{array}\right), \\
& F=\left(\sigma_{1} \psi_{1},\left(1-\sigma_{1}\right) \psi_{1}, 0,0,0,0, \sigma_{2} \psi_{3},\left(1-\sigma_{2}\right) \psi_{3}, 0,0,0, \psi_{2 s}, \psi_{2 i}, \lambda_{4}\right)^{T} \text {, }
\end{aligned}
$$

where $a_{1}=\left(\alpha_{3}+\mu_{1}+\delta_{1 b}\right), a_{2}=\left(\alpha_{4}+\mu_{1}+\delta_{1 s}\right), a_{3}=\left(\alpha_{5}+\right.$ $\left.\mu_{1}+\delta_{1 p}\right), a_{4}=\left(\gamma_{3}+\mu_{3}+\delta_{3 b}\right), a_{5}=\left(\gamma_{4}+\mu_{3}+\delta_{3 s}\right), g_{1}=$ $\left(\alpha_{1} G_{1}(t)+\mu_{1}\right), g_{2}=\left(\gamma_{1} G_{2}(t)+\mu_{3}\right)$, and $g_{3}=\left(\beta G_{3}(t)+\mu_{2}\right)$.

Now from submatrices $A_{11}, A_{12}, A_{21}$, and $A_{22}$ we can deduce that matrix $A(x)$ is a Metzler matrix such that all of its off-diagonal elements are nonnegative, $\forall x \in \mathbb{R}_{+}^{14}$, and $F \geq 0$ is Lipschitz continuous. Thus the feasible region for the model system ((8a), (8b), (8c), (8d), (8e), (8f))-(11) is the set

$$
\Phi=\left\{\left(S_{H}, E_{H}, I_{H B}, I_{H S}, I_{H P}, R_{H}, S_{R}, E_{R}, I_{R B}, I_{R S}, I_{R P}, S_{F}, I_{F},\right.\right.
$$

$$
\text { A) } \left.\geq 0 \in \mathbb{R}_{+}^{14}\right\} \text {. }
$$

This means that any trajectory of the system starting from an initial state in the positive orthant of $\mathbb{R}_{+}^{14}$ remains forever in $\Phi$.

3.2. Positivity of the Solution. We need to show that all variables and parameters of the model are nonnegative, $\forall t \geq$ 0 . We now solve the equations of the system in their patches for testing the positivity. We found that, by letting the initial values of the systems ((8a), (8b), (8c), (8d), (8e), (8f)), ((9a), (9b), (9c), (9d), (9e)), ((10a), (10b)), and (11) be $S_{H}(0)>0$, $S_{R}(0)>0, S_{F}(0)>0$ and $A_{0} \geq 0 E_{H}(0) \geq 0, I_{H B}(0) \geq 0$, $I_{H S}(0) \geq 0, I_{H P}(0) \geq 0, R_{H}(0) \geq 0, E_{R}(0) \geq 0, I_{R B}(0) \geq 0$, $I_{R S}(0) \geq 0, I_{R P}(0) \geq 0$, and $I_{F}(0) \geq 0$, in the solution 
set $S_{H}(t), S_{R}(t), S_{F}(t), A(t), E_{H}(t), I_{H B}(t), I_{H S}(t), I_{H P}(t)$, $R_{H}(t), E_{R}(t), I_{R B}(t), I_{R S}(t), I_{R P}(t)$, and $I_{F}(t)$ are nonnegative, $\forall t \geq 0$.

\section{Model Analysis}

4.1. Disease-Free Equilibrium Solution. The periodic model system ((8a), (8b), (8c), (8d), (8e), (8f))-(11) with nonnegative, continuous periodic functions has disease-free equilibrium solution in which we consider the following equations:

$$
\begin{aligned}
& \frac{d S_{H}}{d t}=\sigma_{1} \psi_{1}-\mu_{1} S_{H} \\
& \frac{d S_{R}}{d t}=\sigma_{2} \psi_{3}-\mu_{3} S_{R} \\
& \frac{d S_{F}}{d t}=\psi_{2 s}-\mu_{2} S_{F} .
\end{aligned}
$$

Now given initial conditions $S_{H}=S_{H 0} \in \mathbb{R}_{+}, S_{R}=S_{R 0} \in$ $\mathbb{R}_{+}$, and $S_{F}=S_{F 0} \in \mathbb{R}_{+}$for (17), (18), and (19), respectively, we will have

$$
\begin{aligned}
& S_{H}=\frac{\sigma_{1} \psi_{1}}{\mu_{1}}+\left(S_{H 0}-\frac{\sigma_{1} \psi_{1}}{\mu_{1}}\right) e^{-\mu_{1} t}, \\
& S_{R}=\frac{\sigma_{2} \psi_{3}}{\mu_{3}}+\left(S_{R 0}-\frac{\sigma_{2} \psi_{3}}{\mu_{3}}\right) e^{-\mu_{3} t}, \\
& S_{R}=\frac{\psi_{2 s}}{\mu_{2}}+\left(S_{F 0}-\frac{\psi_{2 s}}{\mu_{2}}\right) e^{-\mu_{2} t} .
\end{aligned}
$$

Ast $\rightarrow \infty$, (17), (18), and (19) admit unique solution $S_{H} \equiv$ $\sigma_{1} \psi_{1} / \mu_{1}, S_{R} \equiv \sigma_{2} \psi_{3} / \mu_{3}$, and $S_{F} \equiv \psi_{2 s} / \mu_{2}$, respectively, which is globally attractive in $\mathbb{R}_{+}^{3}$.

To find the disease-free equilibrium point we set the derivatives of system ((8a), (8b), (8c), (8d), (8e), (8f))-(11) equal to zero. Then the model system has disease-free solution which is obtained by setting $I_{H B}=I_{H S}=I_{H P}=$ $E_{H}=R_{H}=0, I_{R B}=I_{R S}=I_{R P}=E_{R}=0, I_{F}=0$, and $A=0$ for human, rodent, flea, and pathogen system, respectively. Hence system ((8a), (8b), (8c), (8d), (8e), (8f))-(11) has a disease-free equilibrium point

$$
\begin{gathered}
E^{0}\left(S_{H}^{0}, E_{H}^{0}, I_{H B}^{0}, I_{H S}^{0}, I_{H P}^{0}, R_{H}^{0}, S_{R}^{0}, E_{R}^{0}, I_{R B}^{0}, I_{R S}^{0}, I_{R P}^{0}, S_{F}^{0}, I_{F}^{0},\right. \\
\left.A^{0}\right)=\left(\frac{\sigma_{1} \psi_{1}}{\mu_{1}}, 0,0,0,0,0, \frac{\sigma_{2} \psi_{3}}{\mu_{3}}, 0,0,0,0, \frac{\psi_{2 s}}{\mu_{2}}, 0,0\right) .
\end{gathered}
$$

\section{Basic Reproduction Number}

Let $\left(\mathbb{R}^{k}, \mathbb{R}^{k}\right)$ be the standard ordered $k$-dimensional Euclidean space with a norm $\|\cdot\|$. For $u, v \in \mathbb{R}^{k}$ we write $u \geq v$ provided $u-v \in \mathbb{R}_{+}^{k}, u>v$ provided $u-v \in \mathbb{R}_{+}^{k} \backslash\{0\}$, and $u \gg v$ if $u-v \in \operatorname{int}\left(\mathbb{R}_{+}^{k}\right)$.

Now let $A(t)$ be the continuous, cooperative, irreducible, and $T$-periodic $k \times k$ matrix function with period $T>0$,
$\Phi_{A(\cdot)}(t)$ be the fundamental solution matrix of the linear ordinary differential system

$$
\frac{d x}{d t}=A(t) x
$$

and $\rho\left(\Phi_{A(\cdot)}(T)\right)$ be the spectral radius of $\Phi_{A(\cdot)}(T)$. By Aronsson and Kellogg [45] it follows that $\Phi_{A(\cdot)}(t)$ is a matrix with all elements positive for each $t>0$. By the Perron Frobenius theorem, $\rho\left(\Phi_{A(\cdot)}(T)\right)$ is the principal eigenvalue of $\Phi_{A(\cdot)}(t)$ in the sense that it is simple and admits an eigenvector $v^{*} \gg 0$. The following result is important for our subsequent comparison argument.

Proposition 1. let $\iota=(1 / T) \ln \left(\rho\left(\Phi_{A}(T)\right)\right)$, and then there exists a positive, T-periodic function $v(t)$ such that $e^{i t} v(t)$ is a solution of $x^{\prime}=A(t) x$.

Proof. Let $v^{*} \gg 0$ be the eigenvector associated with the spectral radius $\rho \Phi_{A(\cdot)}(T)$.

By the change of variable

$$
x(t)=e^{\mu t} v(t)
$$

system (22) becomes

$$
\frac{d v}{d t}=A(t) v-\mu v=(A(t)-\mu I) v
$$

where $I$ is an identity matrix.

Thus $v(t)=\Phi_{(A(\cdot)-\mu I)}(t) v^{*}$ is a positive solution of (24). We can easily see that

$$
e^{\mu t} \Phi_{(A(\cdot)-\mu I)}(t)=\Phi_{A(\cdot)}(t) .
$$

Moreover

$$
\begin{aligned}
v(T) & =\Phi_{(A(\cdot)-\mu I)}(t) v^{*}=e^{-\mu T} \Phi_{A(\cdot)}(T) v^{*} \\
& =e^{-\mu T} \rho\left(\Phi_{A(\cdot)}(T)\right) v^{*}=v^{*}=v(0) .
\end{aligned}
$$

Thus, $v(t)$ is a positive $T$-periodic solution of (24) and hence, $x(t)=e^{\mu t} v(t)$ is a solution of (22).

The plague disease model system ((8a), (8b), (8c), (8d), (8e), (8f))-(11) has unique disease-free equilibrium point given in (21).

We consider a heterogeneous population whose individuals are distinguishable by stage of the disease and hence identifiable and put into epidemiological compartments which are $S_{H}, E_{H}, I_{H B}, I_{H S}, I_{H P}, R_{H}, S_{R}, E_{R}, I_{R B}, I_{R S}, I_{R P}, S_{F}, I_{F}$, and $A$. We sort the compartments so that the first $m$ compartments correspond to infected individuals.

We now let

$\mathscr{F}_{i}(x)$ be the rate of appearance of new infections in the $i$ th compartments;

$\mathscr{V}_{i}^{+}(x)$ be the rate of transfer of individuals into compartment $i$ by all other means, other than the epidemic ones;

$\mathscr{V}_{i}^{-}(x)$ be the rate of transfer of individuals out of compartment $i$. 
Then the plague disease transmission model in ((8a), (8b), (8c), (8d), (8e), (8f))-(11) is governed by a periodic ordinary differential system given in

$$
\frac{d x_{i}}{d t}=\mathscr{F}_{i}(t, x)-\mathscr{V}_{i}(t, x) \triangleq f_{i}(t, x)
$$

where $\mathscr{V}_{i}(x)=\mathscr{V}_{i}^{-}(x)-\mathscr{V}_{i}^{+}(x)$.

We rearrange the system by sorting the infectious classes $\left(E_{H}, I_{H B}, I_{H S}, I_{H P}, E_{R}, I_{R B}, I_{R S}, I_{R P}, I_{F}, A\right)$ coming first. We then have

$$
\mathscr{F}(x)=\left(\begin{array}{c}
\left(1-\sigma_{1}\right) \psi_{1}+\alpha_{1} \overline{G_{1}(t)} S_{H} \\
0 \\
0 \\
0 \\
\left(1-\sigma_{2}\right) \psi_{3}+\gamma_{1} \overline{G_{2}(t)} S_{R} \\
0 \\
0 \\
0 \\
\psi_{2 i}+\beta \overline{G_{3}(t)} S_{R} \\
0
\end{array}\right),
$$

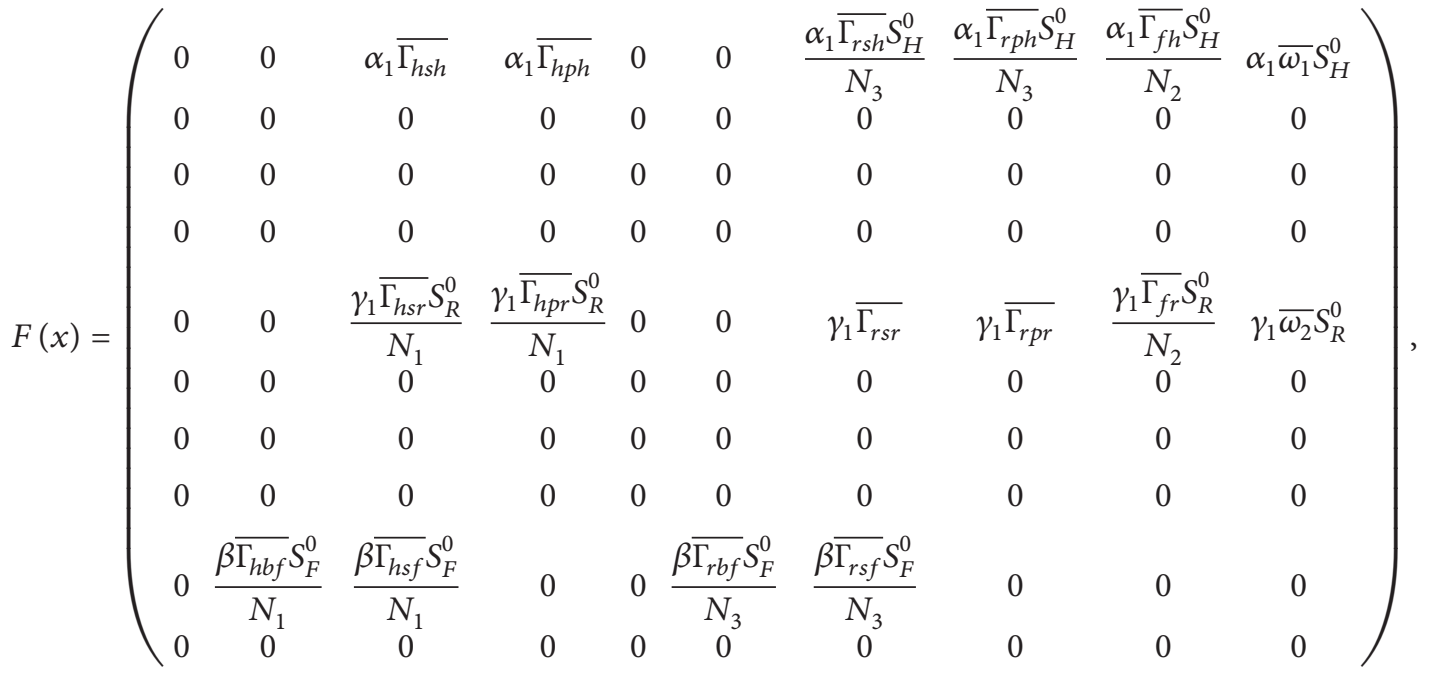

$$
\begin{aligned}
& V(x)=\left(\begin{array}{ll}
v_{11} & v_{12} \\
v_{21} & v_{22}
\end{array}\right),
\end{aligned}
$$

$\mathscr{V}(x)$

$$
=\left(\begin{array}{c}
\alpha_{2} E_{H}+\mu_{1} E_{H} \\
\left(\alpha_{3}+\mu_{1}+\delta_{1 b}\right) I_{H B}-\alpha_{2} \nu_{2} E_{H} \\
\left(\alpha_{4}+\mu_{1}+\delta_{1 s}\right) I_{H S}-\alpha_{3} \rho_{3} I_{H B}-\alpha_{2} \nu_{3} E_{H} \\
\left(\alpha_{5}+\mu_{1}+\delta_{1 p}\right) I_{H P}-\alpha_{2} \nu_{1} E_{H}-\alpha_{3} \rho_{1} I_{H B}-\alpha_{4} \xi I_{H S} \\
\gamma_{2} E_{R}+\mu_{3} E_{R} \\
\left(\gamma_{3}+\mu_{3}+\delta_{3 b}\right) I_{R B}-\gamma_{2} \tau_{3} E_{R} \\
\left(\gamma_{4}+\mu_{3}+\delta_{3 s}\right) I_{R S}-\gamma_{2} \tau_{2} E_{R}-\gamma_{3}(1-\theta) I_{R B} \\
\left(\mu_{3}+\delta_{3 p}\right) I_{R P}-\gamma_{2} \tau_{1} E_{r}-\gamma_{3} \theta I_{R B}-\gamma_{4} I_{R S} \\
\left(\mu_{2}+\delta_{2}\right) I_{F} \\
\eta_{1}(t) I_{H P}-\frac{\eta_{2}(t) I_{R P}}{N_{3}}+\lambda_{4}
\end{array}\right) .
$$

Then we have

$$
\begin{aligned}
F(t) & =\left(\frac{\partial \mathscr{F}_{i}}{\partial x_{j}}\left(x_{0}\right)\right), \\
V(t) & =\left(\frac{\partial \mathscr{V}_{i}}{\partial x_{j}}\left(x_{0}\right)\right),
\end{aligned}
$$

with $1 \leq i, j \leq 10$.

Now using (30) the matrices $F$ and $V$ are as given below:

$\left.\begin{array}{cccc}\frac{\alpha_{1} \overline{\Gamma_{r s h}} S_{H}^{0}}{N_{3}} & \frac{\alpha_{1} \overline{\Gamma_{r p h}} S_{H}^{0}}{N_{3}} & \frac{\alpha_{1} \overline{\Gamma_{f h}} S_{H}^{0}}{N_{2}} & \alpha_{1} \overline{\omega_{1}} S_{H}^{0} \\ 0 & 0 & 0 & 0 \\ 0 & 0 & 0 & 0 \\ 0 & 0 & 0 & 0 \\ \gamma_{1} \overline{\Gamma_{r s r}} & \gamma_{1} \overline{\Gamma_{r p r}} & \frac{\gamma_{1} \overline{\Gamma_{f r}} S_{R}^{0}}{N_{2}} & \gamma_{1} \overline{\omega_{2}} S_{R}^{0} \\ 0 & 0 & 0 & 0 \\ 0 & 0 & 0 & 0 \\ 0 & 0 & 0 & 0 \\ \frac{\beta \overline{\Gamma_{r s f}} S_{F}^{0}}{N_{3}} & 0 & 0 & 0 \\ 0 & 0 & 0 & 0\end{array}\right)$,

where

$$
V_{11}=\left(\begin{array}{ccccc}
\alpha_{2}+\mu_{1} & 0 & 0 & 0 & 0 \\
-\alpha_{2} \nu_{2} & \alpha_{3}+\mu_{1}+\delta_{1 b} & 0 & 0 & 0 \\
-\alpha_{2} \nu_{3} & -\alpha_{3} \rho_{3} & \alpha_{4}+\mu_{1}+\delta_{1 s} & 0 & 0 \\
-\alpha_{2} \nu_{1} & -\alpha_{3} \rho_{1} & -\alpha_{4} \xi & \alpha_{5}+\mu_{1}+\delta_{1 p} & 0 \\
0 & 0 & 0 & 0 & \gamma_{2}+m u_{3}
\end{array}\right),
$$




$$
\begin{aligned}
& V_{22}=\left(\begin{array}{ccccc}
\gamma_{3}+\mu_{3}+\delta_{3 b} & 0 & 0 & 0 & 0 \\
-\gamma_{3}(1-\theta) & \gamma_{4}+\mu_{3}+\delta_{3 s} & 0 & 0 & 0 \\
-\gamma_{3} \theta & -\gamma_{4} & \mu_{3}+\delta_{3 p} & 0 & 0 \\
0 & 0 & 0 & \mu_{2}+\delta_{2} & 0 \\
0 & 0 & -\frac{\eta_{2}}{N_{3}} & 0 & \mu_{4}
\end{array}\right) \\
& V_{21}=\left(\begin{array}{ccccc}
0 & 0 & 0 & 0 & -\gamma_{2} \tau_{3} \\
0 & 0 & 0 & 0 & -\gamma_{2} \tau_{2} \\
0 & 0 & 0 & 0 & -\gamma_{2} \tau_{1} \\
0 & 0 & 0 & 0 & 0 \\
0 & 0 & 0 & -\frac{\eta_{1}}{N_{1}} & 0
\end{array}\right) \\
& V_{12}=\left(\begin{array}{ccccc}
0 & 0 & 0 & 0 & 0 \\
0 & 0 & 0 & 0 & 0 \\
0 & 0 & 0 & 0 & 0 \\
0 & 0 & 0 & 0 & 0 \\
0 & 0 & 0 & 0 & 0
\end{array}\right)
\end{aligned}
$$

Following the setting by Wang and Zhao [46] and van den Driessche and Watmough [47] for epidemic models we check conditions (A1)-(A7) for plague disease epidemic model. System ((8a), (8b), (8c), (8d), (8e), (8f))-(11) is equivalent to periodic ordinary differential system (27). Now considering this system we can easily see that conditions (A1)-(A5) stated below are satisfied.

(A1) Since each function represents a directed transfer of individuals (human, rodent, flea, and pathogens in the environment), they are all nonnegative. Thus, for each $1 \leq i \leq n$ the functions $\mathscr{F}_{i}(t, x), \mathscr{V}_{i}^{+}(t, x)$, and $\mathscr{V}_{i}^{-}(t, x)$ are nonnegative and continuous on $\mathbb{R} \times \mathbb{R}_{+}^{n}$ and continuously differentiable with respect to $x$.

(A2) There is a real number $T>0$ such that for each $1 \leq$ $i \leq n$ the functions $\mathscr{F}_{i}(t, x), \mathscr{V}_{i}^{+}(t, x)$, and $\mathscr{V}_{i}^{-}(t, x)$ are $T$-periodic in $t$.

(A3) If a compartment is empty, there will be no transfer of individuals out of the compartment by any means. That is to say, if $x_{i}=0$ then $\mathscr{V}_{i}^{-}=0$. In particular if $x \in X_{s}$, then $\mathscr{V}_{i}^{-}=0$ for $i=1, \ldots, m$.

(A4) The incidence of infection for uninfected compartments is zero. That is to say, $\mathscr{F}_{i}=0$ for $i>m$.

(A5) If the population is disease-free then the population will remain free of disease. Thus if $x \in X_{s}$, then $\mathscr{F}_{i}=$ 0 and $\mathscr{V}_{i}^{+}=0$ for $i=1, \ldots, m$.

We know that system (27) has disease-free periodic solution given in (21). Now we define $f(t, x(t))=$ $\mathscr{F}(t, x(t))-\mathscr{V}(t, x(t))$ and $M(t)=\left(\partial f_{i}\left(t_{i}, E^{0}\right) / \partial x_{j}\right)$, $11 \leq i, j \leq 14$, where $f_{i}(t, x(t))$ and $x_{i}$ are the $i$ th components of $f(t, x(t))$ and $x$, respectively. Now from (28) and (29) we obtain a $4 \times 4$ matrix given in

$$
\mathbf{A}(\mathbf{t})=\left(\begin{array}{cccc}
-\mu_{1} & \omega & 0 & 0 \\
0 & -\left(\omega+\mu_{1}\right) & 0 & 0 \\
0 & 0 & -\mu_{3} & 0 \\
0 & 0 & 0 & -\mu_{2}
\end{array}\right) .
$$

We then let $\Phi_{A(\cdot)}(t)$ be the monodromy matrix of the linear $T$-periodic system $d z / d t=A(t) z$. Then $\rho \Phi_{A(\cdot)}(T)<1$ which implies that $E^{0}$ is linearly asymptotically stable in the disease-free subspace $X_{s}=\left\{x \geq 0: x_{i}=0, \forall i=1 \cdots m\right\}$, where $i=1 \cdots m$ are the infected compartments. Thus condition (A6) stated below holds.

(A6) The disease-free periodic solution is asymptotically stable in a disease-free subspace $X_{s}$; that is, $\rho \Phi_{A(\cdot)}(T)<1$, where $\rho \Phi_{A(\cdot)}(T)$ is the principal eigenvalue of $\Phi_{A(\cdot)}(t)$.

Next we set $F(t)$ and $V(t)$ as two $10 \times 10$ matrices defined by (30); then using (28) and (29) we get matrices $F(t)$ and $V(t)$ given in (31) and (32), respectively. We can further see that matrix $F(t)$ is nonnegative, and $-V(t)$ is cooperative in the sense that the offdiagonal elements are nonnegative. Let $Y(t, s), t \geq s$, be the evolution operator of our $T$-periodic system

$$
\frac{d y}{d t}=-V(t) y
$$


That is, for each $s \in \mathbb{R}$ the $10 \times 10$ matrix $Y(t, s)$ satisfies

$$
\frac{d Y(t, s)}{d t}=-V(t) Y(t, s), \quad \forall t \geq s, Y(s, s)=I d,
$$

where $I d$ is a $10 \times 10$ identity matrix. Thus the monodromy matrix $\Phi_{V(t)}$ of (35) equals $Y(t, 0), t \geq 0$. Therefore condition (A7) stated below holds.

(A7) The evolution of individuals in the infectious compartments decays exponentially due to natural and disease induced mortalities. Thus $\rho \Phi_{V}(T)<1$.

Now using the standard theory of linear periodic system by Hale [48], there exist $K>0$ and $\wp>0$ such that

$$
\|Y(t, s)\| \leq K e^{-\wp(t-s)}, \quad \forall t \geq s, s \in \mathbb{R} .
$$

We then have

$$
\begin{array}{r}
\|Y(t, t-a) F(t-a)\| \leq K\|F(t-a)\| e^{-\wp a}, \\
\forall t \in \mathbb{R}, a \in[0, \infty) .
\end{array}
$$

Considering the periodic environment we suppose that $\Phi(s), T$-periodic in $s$, is the distribution of the new infection at a rate $F(s) \Phi(s)$ produced by the infected individuals who were introduced at time $s$. Given $t \geq s$ then $Y(t, s) F(s) \Phi(s)$ yields the distribution of those infected individuals who were newly infected at time $s$ and remain in the infected class at $t$. We then have

$$
\begin{aligned}
\Psi(t) & =\int_{-\infty}^{t} Y(t, s) F(s) \Phi(s) d s \\
& =\int_{0}^{\infty} Y(t, t-a) F(t-a) \Phi(t-a) d a,
\end{aligned}
$$

which is the distribution of accumulative new infections at time $t$ produced by all those infected individual $\Phi(s)$ introduced at previous time $s$ to $t(s \leq t)$.

Let $C_{T}$ be the ordered Banach space of all $T$-periodic function from $\mathbb{R}$ to $\mathbb{R}^{n}$, which is equipped with the maximum norm $\|\cdot\|_{\infty}$ and the positive cone $C_{T}^{+}=\left\{\Phi \in C_{T} \Phi(t) \geq 0, t \in\right.$ $\mathbb{R}\}$. Define a linear operator $L: C_{T} \rightarrow C_{T}$ by

$$
\begin{aligned}
&(L \Phi)(t)=\int_{0}^{\infty} Y(t, t-a) F(t-a) \Phi(t-a) d a, \\
& \forall t \in \mathbb{R}, \Phi \in C_{T} .
\end{aligned}
$$

Now by Wang and Zhao [46], Diekmann et al. [49], and van den Driessche and Watmough [47] we name $L$ as the next infection operator; then the basic reproduction number $R_{T}$ of the periodic system $((8 \mathrm{a}),(8 \mathrm{~b}),(8 \mathrm{c}),(8 \mathrm{~d}),(8 \mathrm{e}),(8 \mathrm{f}))-(11)$ is given:

$$
R_{T}=\rho(L),
$$

where $\rho(L)$ is the spectral radius of $L$.
5.1. Characterization of $R_{T}$. In this subsection we investigate whether the basic reproduction number in our periodic system can characterize the threshold of the disease invasion. To do this we consider the following linear $T$-periodic equation:

$$
\frac{d w}{d t}=\left[-V(t)+\frac{F(t)}{\lambda}\right] w, \quad \forall t \in \mathbb{R},
$$

with parameter $\lambda \in(0, \infty)$. Let $W(t, s, \lambda), t \geq s, s \in \mathbb{R}$, be the evolution operator of system $(42)$ on $\mathbb{R}^{10}$. We can clearly see that $\Phi_{F-V}(t)=W(t, 0,1), \forall t \geq 0$. Considering matrices (31) and (32) we note that, for each $\lambda \in(0, \infty)$, all offdiagonal elements of matrix $-V(t)+F(t) / \lambda$ are nonnegative (cooperative matrix). It follows that the linear operator $W(t, s, \lambda)$ is positive in $\mathbb{R}^{10}$ for each $t \geq s, s \in \mathbb{R}$. Now using Perron-Frobenius theorem by Smith and Waltman [50] it entails that $\rho(W(T, 0, \lambda))$ is an eigenvalue of $W(T, 0, \lambda)$ with a nonnegative eigenvector. Also using matrix similarity concept by Shores [51] we can easily verify that matrix $W(s+$ $T, 0, \lambda)$ is similar to the matrix $W(T, 0, \lambda)$ and hence $\sigma(W(s+$ $T, 0, \lambda))=\sigma(W(T, 0, \lambda))$ for any $s \in \mathbb{R}$, where $\sigma(D)$ is a spectrum of the matrix $D$.

Proposition 2 (see [46]). We let (A1)-(A7) hold for system ((8a), (8b), (8c), (8d), (8e), (8f))-(11); then

(i) if $\rho(W(T, 0, \lambda))=1$ has a positive solution $\lambda_{0}$, then $\lambda_{0}$ is an eigenvalue of $L$, and hence $R_{T}>0$;

(ii) if $R_{T}>0$, then $\lambda=R_{T}$ is the unique solution of $\rho(W(T, 0, \lambda))=1$;

(iii) $R_{T}=0$ if and only if $\rho(W(T, 0, \lambda))<1 \forall \lambda>0$.

This result shows that, in order to find the basic reproduction number, we need to find the monodromy matrix $\Phi_{F-V}(t)$ of system (42) and evaluate it. We then find the spectral radius of $\Phi_{F-V}(T)$ and solve the equation $\rho\left(\Phi_{F-V}(T)\right)=1$ for $\lambda$ which is the basic reproduction number.

5.2. Computation of the Basic Reproduction Number. We compute a time-averaged basic reproduction number $R_{0}$ using the next-generation matrix as outlined by Wesley and Allen [52], Heesterbeek [53], and Diekmann et al. [49]. The method has the advantage over the usual next-generation method in that the steps to reach an estimate of $R_{0}$ and the matrix elements of the next-generation matrix have a clear biological basis. It is easy to handle complex diseases like plague disease which has multiple transmission roots from different infectious agents.

To do this we first categorize individuals by their state at the moment they become infected (type at infection). These types at infection refer specifically to the birth of the infection in the individual. These categories (types at infection) differ in the way they transmit plague disease which in turn differentiates their ability to produce secondary cases.

In our case we categorize the individuals into eight states and label them as follows: human infected with bubonic plague (type 1), human infected with septicemic plague (type 
2), human infected with pneumonic plague (type 3), rodent infected with bubonic plague (type 4), rodent infected with septicemic plague (type 5), rodent infected with pneumonic plague (type 6), flea infested with pathogens (type 7), and the pathogens in the environment (type 8).

We assume and label individual with bubonic plague as stage one of the disease, septicemic plague as stage two, and pneumonic plague as stage three. We also assume that when an individual in stage one graduates to stage two we only consider the current stage and ignore the latter. We assume that the infection only goes in ascending direction that is from stages one to two, or two to three, but not in the reverse direction.

Since the system has eight types at infection, the nextgeneration matrix, $\mathbf{K}$, will be an $8 \times 8$ matrix with elements $k_{i j}$ 's. Each of the elements $k_{i j}$ 's stands for expected number of new cases of $i$ caused by one infected individual of $j$. For example, $k_{11}$ is the expected number of new cases of humans infected with bubonic plague caused by one infected human with bubonic plague.

We now define a matrix $\mathbf{K}$ whose entries are $k_{i j}$. The resulting next-generation matrix is as given in

$$
\mathbf{K}=\left(\begin{array}{llllllll}
k_{11} & k_{12} & k_{13} & k_{14} & k_{15} & k_{16} & k_{17} & k_{18} \\
k_{21} & k_{22} & k_{23} & k_{24} & k_{25} & k_{26} & k_{27} & k_{28} \\
k_{31} & k_{32} & k_{33} & k_{34} & k_{35} & k_{36} & k_{37} & k_{38} \\
k_{41} & k_{42} & k_{43} & k_{44} & k_{45} & k_{46} & k_{47} & k_{48} \\
k_{51} & k_{52} & k_{53} & k_{54} & k_{55} & k_{56} & k_{57} & k_{58} \\
k_{61} & k_{62} & k_{63} & k_{64} & k_{65} & k_{66} & k_{67} & k_{68} \\
k_{71} & k_{72} & k_{73} & k_{74} & k_{75} & k_{76} & k_{77} & k_{78} \\
k_{81} & k_{82} & k_{83} & k_{84} & k_{85} & k_{86} & k_{87} & k_{88}
\end{array}\right) .
$$

Then, $R_{0}=\rho(K)$, where $\rho(K)$ is spectral radius of $K$.

Some elements equal 0 because not all types of infections cause all other types of infection. For example, humans with bubonic plague $I_{H B}$ (type at infection 1) do not produce type at infections 1 (human infected with bubonic plague), 4 (rodent infected with bubonic plague), 5 (rodent infected with septicemic plague), 6 (rodent infected with pneumonic plague), and 8 (pathogens in the environment). This means that $k_{11}, k_{14}, k_{15}, k_{16}$, and $k_{18}$ are 0 . The type at infection
2 (human infected with septicemic plague) also does not produce type at infections 1 (human infected with bubonic plague), 4 (rodent infected with bubonic plague), 6 (rodent infected with pneumonic plague), and 8 (pathogens in the environment). This also means that $k_{21}, k_{24}, k_{26}$, and $k_{28}$ are zero (0). The type at infection 3 does not produce type at infections 1 (human infected with bubonic plague), 2 (human infected with septicemic plague), 4 (rodent infected with bubonic plague), 5 , and 7 which means that $k_{31}, k_{32}, k_{34}, k_{35}$, and $k_{37}$ are zero. Type at infection 4 does not produce type at infection $1,2,3,4$, or 8 which means that $k_{41}, k_{42}, k_{43}, k_{44}$, and $k_{48}$ are zero. Type at infection 5 does not produce type at infections $1,3,4$, and 8 ; then $k_{51}, k_{53}, k_{54}$, and $k_{58}$ are zero. The type at infection 6 does not produce type at infections 1 , $2,4,5$, and 7 ; thus $k_{61}, k_{62}, k_{64}, k_{65}$, and $k_{67}$ are zero. Type at infection 7 also does not produce type at infections 3, 6, 7 , and 8; thus $k_{73}, k_{76}, k_{77}$, and $k_{78}$ are zero. And the type at infection 8 does not produce type at infections $1,2,4,5$, 7, and 8 which means that $k_{81}, k_{82}, k_{84}, k_{85}, k_{87}$, and $k_{88}$ are zero. Incorporating these, we modify the matrix $\mathbf{K}$ as shown in the following matrix:

$$
\mathbf{K}=\left(\begin{array}{cccccccc}
0 & 0 & 0 & 0 & 0 & 0 & k_{17} & 0 \\
k_{21} & k_{22} & 0 & 0 & k_{25} & 0 & k_{27} & 0 \\
k_{31} & k_{32} & k_{33} & 0 & 0 & k_{36} & 0 & k_{38} \\
0 & 0 & 0 & 0 & 0 & 0 & k_{47} & 0 \\
0 & 0 & 0 & k_{54} & k_{55} & 0 & k_{57} & 0 \\
0 & 0 & 0 & k_{64} & k_{65} & k_{66} & 0 & k_{68} \\
k_{71} & k_{72} & 0 & k_{74} & k_{75} & 0 & 0 & 0 \\
0 & 0 & k_{83} & 0 & 0 & k_{86} & 0 & 0
\end{array}\right) .
$$

We will now explain the derivation of each matrix element in detail. We employ the derivation steps by Gail and Benichou [54] to drive the expressions for $k_{i j}$. We mainly base our derivation on the adequate contact rate between the infected individual type $j$ and the susceptible individual type $i$, the expected duration of infection of individual type $j$, and the probability that the individual type $j$ survives the duration between the latent stage and the time an individual experiences the onset of clinical disease as in

$$
\begin{aligned}
\mathbf{K}_{\mathbf{i j}}= & (\text { Effective contact Rate }) \times(\text { Duration of infection }) \\
& \times(\text { Probability that the individual survive the incubation period }) .
\end{aligned}
$$

The production of $I_{H B}$ depends on the probability that the total number of fleas that become infectious at the rate of $\beta$ and the infected immigrants survive the incubation period. We also consider the rate at which $I_{F}$ adequately bites the susceptible human and the bite results in a human infected with bubonic plague $I_{H B}$ at the average value of transmission rate $\overline{\Gamma_{f h}}$. The total number of humans infected with bubonic plague caused by one flea infested with pathogens is as given in

$$
k_{17}=\left(\frac{\beta}{\beta+\mu_{2}}+\frac{\psi_{2 i}}{\psi_{2 i}+\mu_{2}}\right) \frac{\nu_{2} \overline{\Gamma_{f h}}}{\mu_{2}+\delta_{2}} .
$$

Septicemic plague in human may be produced in various ways: progression of untreated human with bubonic 
plague to human with septicemic plague, adequate contact (including sexual contact) between humans with septicemic plague, adequate contact between rodent and human with septicemic plague, and being acquired from the flea infested with pathogens. We consider the progression rate of infected human with bubonic to septicemic $\alpha_{3} \rho_{3}$, the adequate contact (it may be sexual contact) rate between humans with septicemic plague, rodent infected with septicemic plague, and the infected flea to human with septicemic plague at the average rates $\overline{\gamma_{h s h}}, \overline{\gamma_{r s h}}$, and $\overline{\gamma_{f h}}$. Then the number of humans infected with septicemic plague from all the mentioned infectious agents is as given in

$$
\begin{aligned}
k_{21} & =\frac{\alpha_{2} \alpha_{3} \nu_{2} \rho_{3}}{\left(\alpha_{2} \nu_{2}+\mu_{1}\right)\left(\mu_{1}+\alpha_{3}+\delta_{1 b}\right)}, \\
k_{22} & =\left(\frac{\alpha_{3} \rho_{3}}{\alpha_{3} \rho_{3}+\mu_{1}}+\frac{\alpha_{2} \nu_{3}}{\alpha_{2} \nu_{3}+\mu_{1}}\right) \frac{\overline{\Gamma_{h s h}}}{\left(\alpha_{4}+\mu_{1}+\delta_{1 s}\right)}, \\
k_{25} & =\left(\frac{\gamma_{2} \tau_{2}}{\left(\gamma_{2} \tau_{2}+\mu_{3}\right)}+\frac{\gamma_{3}(1-\phi)}{\gamma_{3}(1-\phi)+\mu_{3}}\right) \\
\cdot & \frac{\left(\gamma_{r s h}+\mu_{3}+\delta_{3 s}\right)}{\left(\frac{\beta}{\beta+\mu_{2}}+\frac{\psi_{2 i}}{\psi_{2 i}+\mu_{2}}\right) \frac{\nu_{1} \overline{\Gamma_{f h}}}{\mu_{2}+\delta_{2}} .}
\end{aligned}
$$

The proportions $\rho_{1}$ and $\xi$ of untreated $I_{H B}$ and $I_{H S}$ may progress and become $I_{H P}$ at the progression rates $\alpha_{3}$ and $\alpha_{4}$, respectively. We multiply the average period $I_{H B}$ remain infected by the rate at which they progress to $I_{H P}$. $I_{H P}$ may also result from the airborne transmission from the human or rodent with pneumonic plague at the average rate $\overline{\gamma_{h p h}}$ or $\overline{\gamma_{r p h}}$, respectively, and through the direct interaction with the environment at the average rate $\overline{\omega_{1}}$. Then the total number of humans infected with pneumonic plague from the stated five sources is given in

$$
\begin{aligned}
k_{31} & =\frac{\alpha_{2} \alpha_{3} \nu_{2} \rho_{1}}{\left(\alpha_{2} \nu_{2}+\mu_{1}\right)\left(\alpha_{3}+\mu_{1}+\delta_{1 b}\right)}, \\
k_{32} & =\left(\frac{\alpha_{3} \rho_{3}}{\alpha_{3} \rho_{3}+\mu_{1}}+\frac{\alpha_{2} \nu_{3}}{\alpha_{2} \nu_{3}+\mu_{1}}\right) \frac{\alpha_{4} \xi}{\alpha_{4}+\mu_{1}+\delta_{1 s}}, \\
k_{33} & =\left(\frac{\alpha_{2} \nu_{1}}{\alpha_{2} \nu_{1}+\mu_{1}}+\frac{\alpha_{3} \rho_{1}}{\alpha_{3} \rho_{1}+\mu_{1}}+\frac{\alpha_{4} \phi}{\alpha_{4} \phi+\mu_{1}}\right) \\
\cdot & \left.\frac{\overline{\Gamma_{h p h}}}{\alpha_{5}+\mu_{1}+\delta_{1 p}}, \overline{\gamma_{4}}\right) \frac{\overline{\Gamma_{r p h}}}{\mu_{3}+\delta_{3 p}} \\
k_{36} & =\left(\frac{\gamma_{2} \tau_{1}}{\gamma_{2} \tau_{1}+\mu_{3}}+\frac{\gamma_{3} \phi}{\gamma_{3} \phi+\mu_{3}}+\frac{\gamma_{4}+\mu_{3}}{\overline{\eta_{2}}}\right) \frac{\overline{\omega_{1}}}{\overline{\mu_{4}}} \\
k_{38} & =\left(\frac{\overline{\lambda_{4}}}{\overline{\lambda_{4}}+\overline{\mu_{4}}}+\frac{\overline{\eta_{1}}}{\overline{\eta_{1}}+\overline{\mu_{4}}}+\frac{\overline{\eta_{2}}+\overline{\mu_{4}}}{}\right.
\end{aligned}
$$

Production of number of rodents with bubonic plague $I_{\mathrm{RB}}$ depends only on the flea infested with pathogens. The infection depends on the infection period of the flea that survives the incubation period and the proportion at which the adequate contact between infected flea and susceptible rodent causes bubonic plague at the average rate $\tau_{3} \overline{\Gamma_{f r}}$ as given in

$$
k_{47}=\left(\frac{\beta}{\beta+\mu_{2}}+\frac{\psi_{2 i}}{\psi_{2 i}+\mu_{2}}\right) \frac{\tau_{3} \overline{\Gamma_{f r}}}{\mu_{2}+\delta_{2}} .
$$

The septicemic plague in rodent is produced in three ways; the first way is when infected rodent with bubonic plague progresses and becomes septicemic plague infective agent at the rate $\gamma_{3}(1-\phi)$. The second way is after adequate contact (it may also be a rodent eating or biting an infected individual) between the susceptible rodent and a rodent infected with septicemic plague or human at the average rate $\overline{\Gamma_{r s r}}$ or $\overline{\Gamma_{h s r}}$, respectively. The third way is from the flea infested with pathogens with the proportion that the adequate contact between $I_{F}$ and the susceptible rodent results in $I_{R S}$. The total number of $I_{R S}$ infected from these infectious agents is as given in

$$
\begin{aligned}
k_{52} & =\left(\frac{\alpha_{3} \rho_{3}}{\alpha_{3} \rho_{3}+\mu_{1}}+\frac{\alpha_{2} \nu_{3}}{\alpha_{2} \nu_{3}+\mu_{1}}\right) \frac{\overline{\Gamma_{h s r}}}{\alpha_{4}+\mu_{1}+\delta_{1 s}}, \\
k_{54} & =\frac{\gamma_{2} \gamma_{3} \tau_{3}(1-\phi)}{\left(\gamma_{2} \tau_{3}+\mu_{3}\right)\left(\gamma_{3}+\mu_{3}+\delta_{3 b}\right)}, \\
k_{55} & \\
= & \left(\frac{\gamma_{2} \tau_{2}}{\left(\gamma_{2} \tau_{2}+\mu_{3}\right)}+\frac{\gamma_{3}(1-\phi)}{\gamma_{3}(1-\phi)+\mu_{3}}\right) \frac{\overline{\Gamma_{r s r}}}{\gamma_{4}+\mu_{3}+\delta_{3 s}}, \\
k_{57} & =\left(\frac{\beta}{\beta+\mu_{2}}+\frac{\psi_{2 i}}{\psi_{2 i}+\mu_{2}}\right) \frac{\tau_{2} \overline{\Gamma_{f r}}}{\mu_{2}+\delta_{2}} .
\end{aligned}
$$

$I_{R P}$ may be the result of airborne transmission between the susceptible rodent and the human and rodent with pneumonic plague at the average rates $\overline{\Gamma_{h p r}}$ and $\overline{\Gamma_{r p r}}$, respectively. It may also occur from the progression of untreated $I_{R B}$ and $I_{R S}$ at the rates $\gamma_{3}$ and $\gamma_{4}$, respectively. The pathogens in environment may also cause $I_{R P}$ after the adequate interaction at the average rate $\overline{\omega_{2}}$. Now the total number of $I_{R B}$ resulting from these interactions is in

$$
\begin{aligned}
k_{63} & =\left(\frac{\alpha_{2} \nu_{1}}{\alpha_{2} \nu_{1}+\mu_{1}}+\frac{\alpha_{3} \rho_{1}}{\alpha_{3} \rho_{1}+\mu_{1}}+\frac{\alpha_{4} \phi}{\alpha_{4} \phi+\mu_{1}}\right) \\
\cdot & \frac{\overline{\Gamma_{h p r}}}{\alpha_{5}+\mu_{1}+\delta_{1 p}}, \\
k_{64} & =\frac{\gamma_{2} \gamma_{3} \tau_{3} \phi}{\left(\gamma_{2} \tau_{3}+\mu_{3}\right)\left(\gamma_{3}+\mu_{3}+\delta_{3 b}\right)},
\end{aligned}
$$




$$
\begin{aligned}
k_{65} & =\left(\frac{\gamma_{2} \tau_{2}}{\left(\gamma_{2} \tau_{2}+\mu_{3}\right)}+\frac{\gamma_{3}(1-\phi)}{\gamma_{3}(1-\phi)+\mu_{3}}\right) \\
\cdot & \frac{\gamma_{4}}{\gamma_{4}+\mu_{3}+\delta_{3 s}}, \\
k_{66} & =\left(\frac{\gamma_{2} \tau_{1}}{\gamma_{2} \tau_{1}+\mu_{3}}+\frac{\gamma_{3} \phi}{\gamma_{3} \phi+\mu_{3}}+\frac{\gamma_{4}}{\gamma_{4}+\mu_{3}}\right) \frac{\overline{\Gamma_{r p r}}}{\mu_{3}+\delta_{3 p}}, \\
k_{68} & =\left(\frac{\lambda_{4}}{\lambda_{4}+\mu_{4}}+\frac{\overline{\eta_{1}}}{\overline{\eta_{1}}+\mu_{4}}+\frac{\overline{\eta_{2}}}{\overline{\eta_{2}}+\mu_{4}}\right) \frac{\overline{\omega_{2}}}{\mu_{4}} .
\end{aligned}
$$

Fleas are infested with pathogens from humans and rodents infected with bubonic and septicemic plague at the average rates $\overline{\gamma_{h b f}}, \overline{\gamma_{h s f}}, \overline{\gamma_{r b f}}$, and $\overline{\gamma_{r s f}}$. The infection is dictated by the probability that humans and rodents with bubonic and septicemic plague survive the incubation period and the adequate rates of contact. From these interactions, we get the total number of infectious fleas, given in

$$
\begin{aligned}
& k_{71}=\frac{\alpha_{2} \nu_{2} \overline{\Gamma_{h b f}}}{\left(\alpha_{2} \nu_{2}+\mu_{1}\right)\left(\mu_{1}+\alpha_{3}+\delta_{1 b}\right)}, \\
& k_{72}=\left(\frac{\alpha_{3} \rho_{3}}{\alpha_{3} \rho_{3}+\mu_{1}}+\frac{\alpha_{2} \nu_{3}}{\alpha_{2} \nu_{3}+\mu_{1}}\right) \frac{\overline{\Gamma_{h s f}}}{\alpha_{4}+\mu_{1}+\delta_{1 s}}, \\
& k_{74}=\frac{\gamma_{2} \tau_{3} \overline{\Gamma_{r b f}}}{\left(\gamma_{2} \tau_{3}+\mu_{3}\right)\left(\gamma_{3}+\mu_{3}+\delta_{3 b}\right)}, \\
& k_{75}=\left(\frac{\gamma_{2} \tau_{2}}{\left(\gamma_{2} \tau_{2}+\mu_{3}\right)}+\frac{\gamma_{3}(1-\phi)}{\gamma_{3}(1-\phi)+\mu_{3}}\right) \frac{\overline{\Gamma_{r s f}}}{\gamma_{4}+\mu_{3}+\delta_{3 s}} .
\end{aligned}
$$

The pathogens are released into the environment at the average rates $\overline{\eta_{1}}$ and $\overline{\eta_{1}}$ from $I_{H P}$ and $I_{R P}$, respectively. The released number of pathogens at a given time depends on the infectious period of the rodent and human infected with pneumonic plague and the probability that $I_{H P}$ and $I_{R P}$ survive the incubation period. The total pathogens in soil/environment is as given in

$$
\begin{aligned}
k_{83} & =\left(\frac{\alpha_{2} \nu_{1}}{\alpha_{2} \nu_{1}+\mu_{1}}+\frac{\alpha_{3} \rho_{1}}{\alpha_{3} \rho_{1}+\mu_{1}}+\frac{\alpha_{4} \xi}{\alpha_{4} \phi+\mu_{1}}\right) \\
& \cdot \frac{\overline{\eta_{1}}}{\alpha_{5}+\mu_{1}+\delta_{1 p}}, \\
k_{86} & =\left(\frac{\gamma_{2} \tau_{1}}{\gamma_{2} \tau_{1}+\mu_{3}}+\frac{\gamma_{3} \phi}{\gamma_{3} \phi+\mu_{3}}+\frac{\gamma_{4}}{\gamma_{4}+\mu_{3}}\right) \frac{\overline{\eta_{2}}}{\mu_{3}+\delta_{3 p}} .
\end{aligned}
$$

Each element in the matrix $\mathbf{K}$ represents the expected number of secondary cases produced by infected individual $j$ during the entire infectious period of that particular individual into a completely susceptible population $i$ [55].

5.2.1. Basic Reproduction Number $R_{0}$. We obtain the average basic reproduction number $R_{0}$ by computing the maximum modulus of the eigenvalues of the next-generation matrix K [49, 53]. Now using Maple computing software package, the basic reproduction number is

$$
\begin{aligned}
R_{0}= & \frac{1}{T} \int_{0}^{T} \frac{k_{22}(s)+k_{55}(s)}{4} \\
& +\frac{1}{2} \sqrt{A_{1}+\frac{1}{3 \sqrt[3]{2}} A_{4}+\frac{A_{5}}{3 A_{4}}} \\
& +\frac{1}{2} \sqrt{A_{2}-\frac{1}{3 \sqrt[3]{2}} A_{4}-\frac{A_{5}}{3 A_{4}}} \\
& +\frac{A_{3}}{4 \sqrt{A_{1}+(1 / 3 \sqrt[3]{2}) A_{4}+A_{5} / 3 A_{4}}} d s,
\end{aligned}
$$

in which

$$
\begin{aligned}
A_{1} & =\frac{3 \vartheta_{3}+8 \vartheta_{1}}{12}, \\
A_{2} & =\frac{3 \vartheta_{3}-8 \vartheta_{1}}{6}, \\
A_{3} & =4 \vartheta_{1} \vartheta_{3}-\vartheta_{3}^{3}-8 \vartheta_{4}, \\
A_{4} & =\frac{1}{3 \sqrt[3]{2}}\left(\left(2 \vartheta_{1}^{3}-72 \vartheta_{2} \vartheta_{1}-9 \vartheta_{3} \vartheta_{4} \vartheta_{1}+27 \vartheta_{4}^{2}\right.\right. \\
& \left.\left.+27 \vartheta_{3}^{2} \vartheta_{2}\right)\right)+\left(\left(2 \vartheta_{1}^{3}-72 \vartheta_{2} \vartheta_{1}-9 \vartheta_{3} \vartheta_{4} \vartheta_{1}+27 \vartheta_{4}^{2}\right.\right. \\
& \left.\left.+27 \vartheta_{3}^{2} \vartheta_{2}^{2}-4\left(\vartheta_{1}^{2}+12 \vartheta_{2}-3 \vartheta_{3} \vartheta_{4}\right)^{3}\right)^{1 / 3}\right)^{1 / 2}, \\
A_{5} & =\sqrt[3]{2}\left(\vartheta_{1}^{2}+12 \vartheta_{2}-3 \vartheta_{3} \vartheta_{4}\right),
\end{aligned}
$$

where

$$
\begin{aligned}
\vartheta_{1} & =k_{22}(s) k_{55}(s)-k_{17}(s) k_{71}(s)-k_{27}(s) k_{72}(s) \\
& -k_{57}(s) k_{75}(s), \\
\vartheta_{2} & =k_{17}(s) k_{55}(s)\left(k_{17}(s) k_{71}(s)+k_{21}(s) k_{72}(s)\right) \\
& -k_{47}(s)\left(k_{25}(s) k_{54}(s) k_{72}(s)\right. \\
& \left.+k_{22}(s)\left(k_{55}(s) k_{74}(s)+k_{54}(s) k_{75}(s)\right)\right), \\
\vartheta_{3} & =-k_{22}(s)-k_{55}(s), \\
\vartheta_{4} & =\left(k_{22}(s)+k_{55}(s)\right)\left(k_{17}(s) k_{71}(s)+k_{47}(s) k_{74}(s)\right) \\
& -k_{72}(s)\left(k_{17}(s) k_{21}(s)-k_{27}(s) k_{55}(s)\right. \\
& \left.+k_{25}(s) k_{57}(s)\right)-k_{75}(s)\left(k_{47}(s) k_{54}(s)\right. \\
& \left.-k_{22}(s) k_{57}(s)\right) .
\end{aligned}
$$

Since the system has multiple infectious types from multiple hosts, then the next-generation matrix produces the average value of the geometric mean of the number of infections per generation and the basic reproduction number 


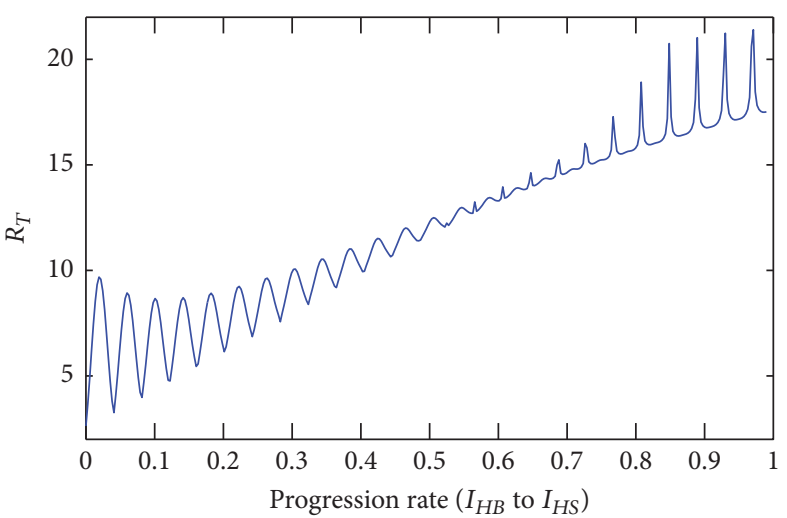

(a)

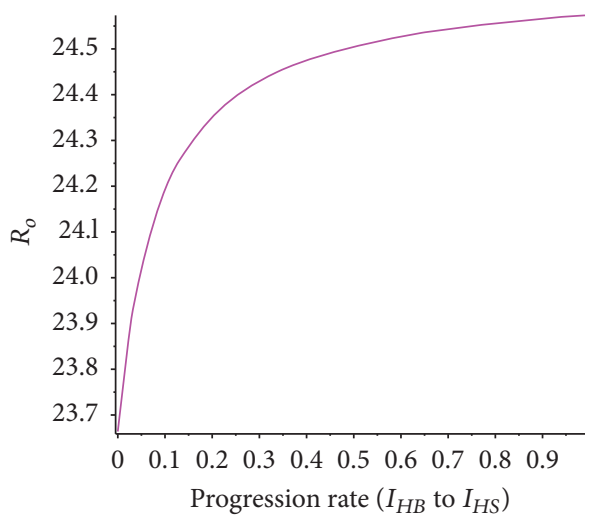

(b)

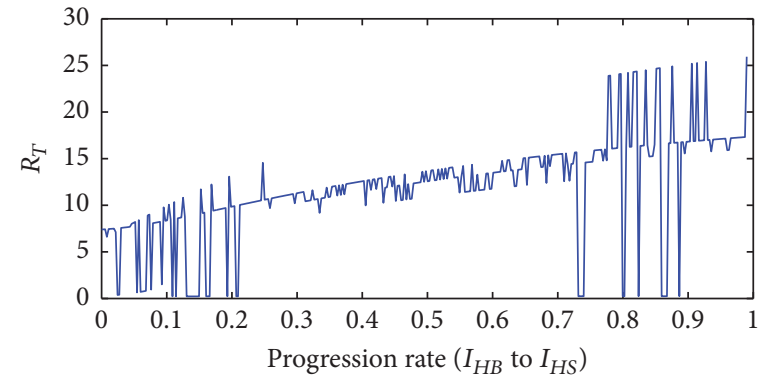

(c)

FIGURE 1: Effect of progression rates from $I_{H B}$ to $I_{H S}$ on the periodic reproduction number.

is the average number of secondary infections [56]. It is shown that the basic reproduction number of plague disease depends on the expected number of new cases of humans infected with bubonic plague caused by one infected flea $\left(k_{17}\right)$, the expected number of new cases of humans infected with septicemic plague caused by one infected human with bubonic plague $\left(k_{21}\right)$, the expected number of new cases of humans infected with septicemic plague caused by one infected human with septicemic plague $\left(k_{22}\right)$, the expected number of new cases of rodents infected with bubonic plague caused by one infected flea $\left(k_{47}\right)$, the expected number of new cases of rodents infected with septicemic plague caused by one infected rodent with bubonic plague $\left(k_{54}\right)$, the expected number of new cases of rodents infected with septicemic plague caused by one infected rodent with septicemic plague $\left(k_{55}\right)$, the expected number of new cases of rodents infected with septicemic plague caused by one infected flea $\left(k_{57}\right)$, the expected number of new cases of fleas infested with Yersinia pestis caused by one infected human with bubonic plague $\left(k_{71}\right)$, the expected number of new cases of fleas infested with Yersinia pestis caused by one infected human with septicemic plague $\left(k_{72}\right)$, the expected number of new cases of fleas infested with Yersinia pestis caused by one infected rodent with bubonic plague $\left(k_{74}\right)$, and the expected number of new cases of fleas infested with Yersinia pestis caused by one infected rodent with septicemic plague $\left(k_{75}\right)$. The result may also be interpreted as follows: among all elements of the matrix $\mathbf{K}, k_{i j}$, which appear in $R_{O}$, gives more significant involvement in the dynamics and spread of plague disease.

\section{Numerical Results and Discussion}

Here we use the parameters values of model system $((8 \mathrm{a})$, (8b), (8c), (8d), (8e), (8f))-(11) given in Table 1 to study the transmission trend of plague disease. Simulation results are given to show the effect of different parameters on the periodic reproduction number. We have also chosen temperature data obtained from Tanga region from January to December 2013 to show the seasonal distribution in the number of secondary cases of plague infections. It is observed that simulation results from time-averaged seasonal parameters and those seasonal parameters treated mathematically as sinusoidal functions match the real seasonal fluctuation data (temperature).

Results shows that the increase in number of individuals infected with bubonic plague, to a large extent, affects the increases in number of individuals with septicemic and pneumonic plague disease. This is due to the fact that individuals with bubonic plague progress and become either septicemic or pneumonic plague infective agents. This in turn leads to the significant increase of plague disease transmission rate and the average number of secondary infections. Figures 2 and 1 show how the progression rates from individuals with bubonic plague to individuals with septicemic plague affect the average number of secondary infections in human beings and rodents, respectively. It is illustrated that the increase of human beings and rodents progressing to become septicemic plague infective agents affects the disease dynamics by increasing the average number of secondary 


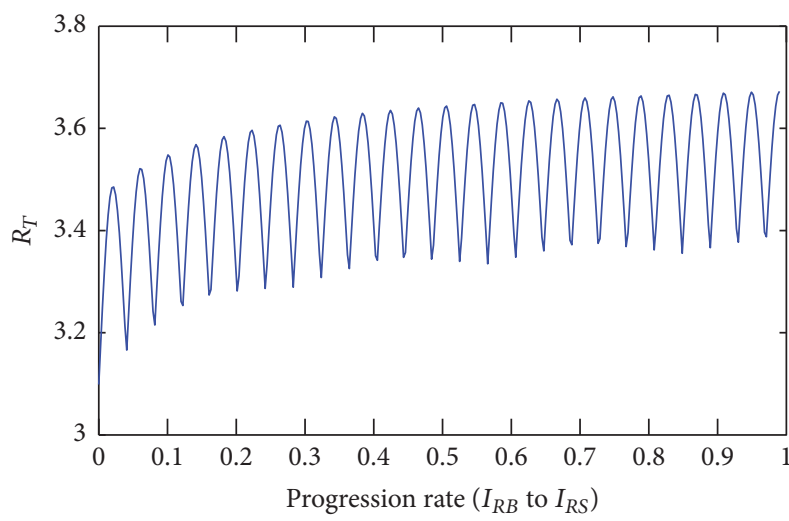

(a)

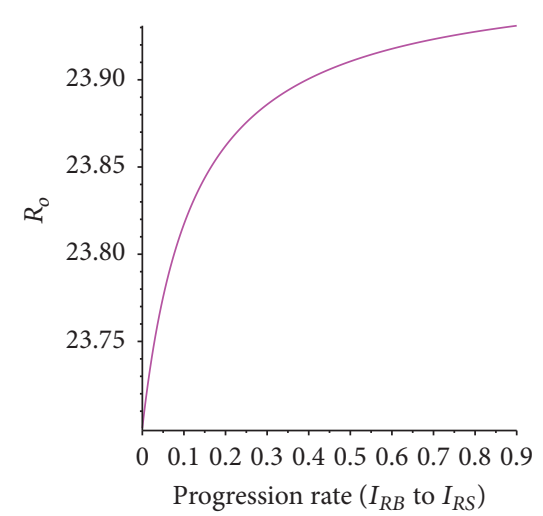

(b)

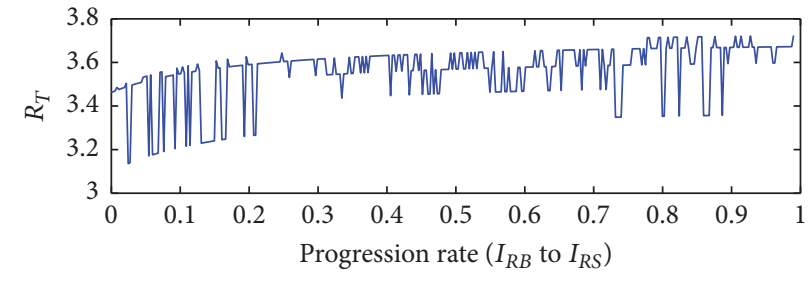

(c)

FIGURE 2: Effect of progression rates from $I_{R B}$ to $I_{R S}$ on the periodic reproduction number.

infections. We see similar result when we evaluate the periodic reproduction number based on the temperature data from Tanga region (Figures 1(c) and 2(c)) and time-averaged parameters (Figures 1(b) and 2(b)) for human beings and rodents, respectively. These findings necessitate the need for early treatment of plague disease infective agents especially the primary forms (bubonic and septicemic plague) before they progress to highly fatal and fast spreading plague forms like pneumonic plague disease. It is thus important for the government and other health stakeholders to ensure the availability of effective plague disease treatment especially in high risk areas.

Increase of plague disease transmission through flea bite in human beings and rodents populations alters the whole dynamics of plague disease. Results in Figures 3 and 4 show the effect of infection from infectious flea to human beings with bubonic and septicemic plague on the average number of secondary infections. The infection from flea to rodents with bubonic and septicemic plague disease also shows the significant effect on $R_{T}$ as illustrated in Figures 5 and 6 , respectively. The results generally show that when the periodic infection rates from flea increase, those of the infectious human beings and rodent increase as well; this in turn affects the general plague disease periodic transmission and spread. These results are in conjuncture with what is observed when $R_{T}$ is evaluated using the temperature data and time-averaged seasonal parameter as in Figures 3(c), 3(b), 5(c), and 5(b) for human beings and rodents, respectively

The results call attention for the need to control the number of infectious fleas and flea population in general as the way of controlling the plague disease. The study recommends that for the appropriate and most effective way to control flea population we first need to study the flea's ecology and its local patterns of disease transmission. One of the most important and cost-effective strategies of controlling the vector flea is environmental management strategies that can reduce or eliminate vector breeding grounds. For example, in residential areas people must be educated to improve their surrounding environment in a way that does not favor the survival and growth of vector flea. This may be through improving the design of water systems, improving waste disposal and water storage, discouraging deforestation and loss of biodiversity, and living in well ventilated housing that is not close to animals.

There are also biological control tools like bacterial larvicides and larvivorous fish that may be used to control flea population [57]. These control methods aim at killing vector larvae without generating the ecological impacts as they do not use chemicals. Another strategy is using chemical methods, which mainly shorten the lifespan of vectors. These tools include indoor residual sprays, space spraying, and use of chemical larvicides and adulticides. Since most of these methods have side effects to the environmental ecology they are recommended to be used when other safe strategies fail. Moreover, even though these chemicals are not environmentally friendly, we advise the environmental stakeholders to recommend the use of chemical methods of vector control that reinforces linkages between health and environment.

Reducing the number of flea population will reduce the infection rates to human beings and rodents and ultimately reduce the number of secondary infections. Figure 7 (a) shows how reducing the number of infectious fleas reduces the number of secondary infections. This is also true when 


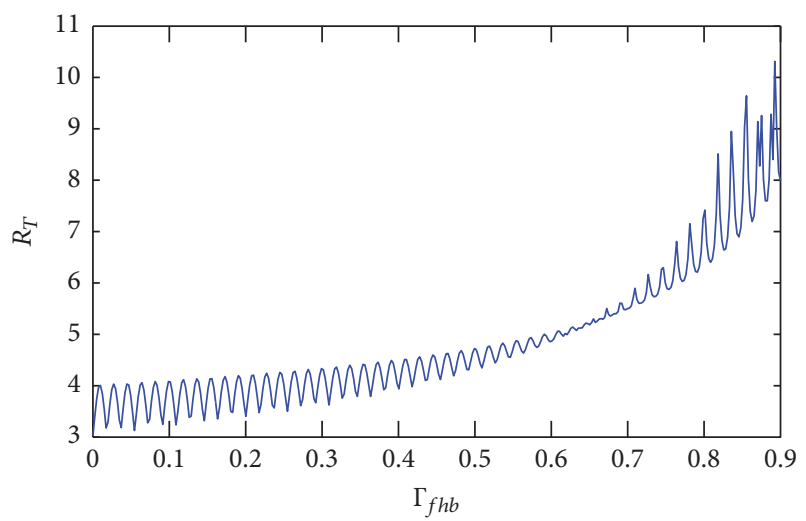

(a)

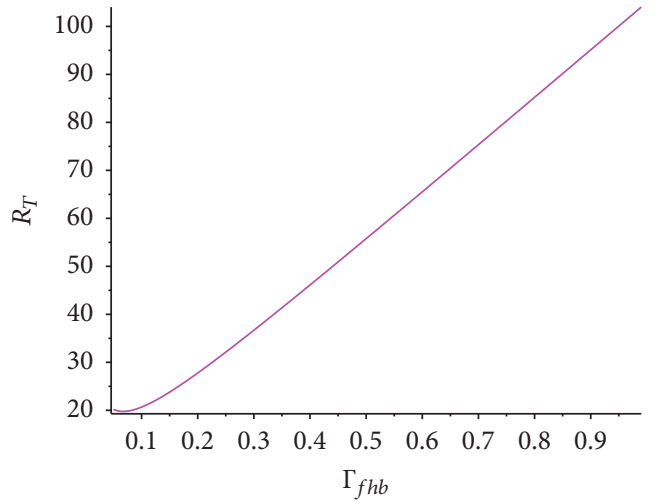

(b)

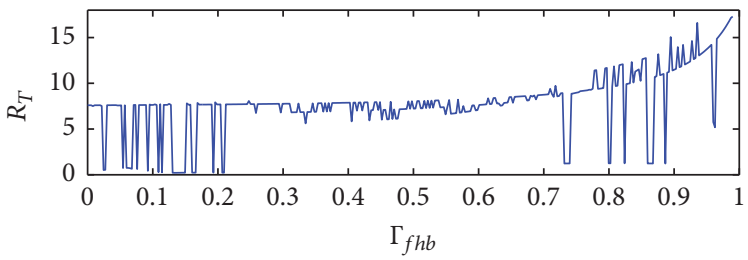

(c)

FIGURE 3: The effect of infection from $I_{F}$ to human beings with bubonic plague on the periodic reproduction number.

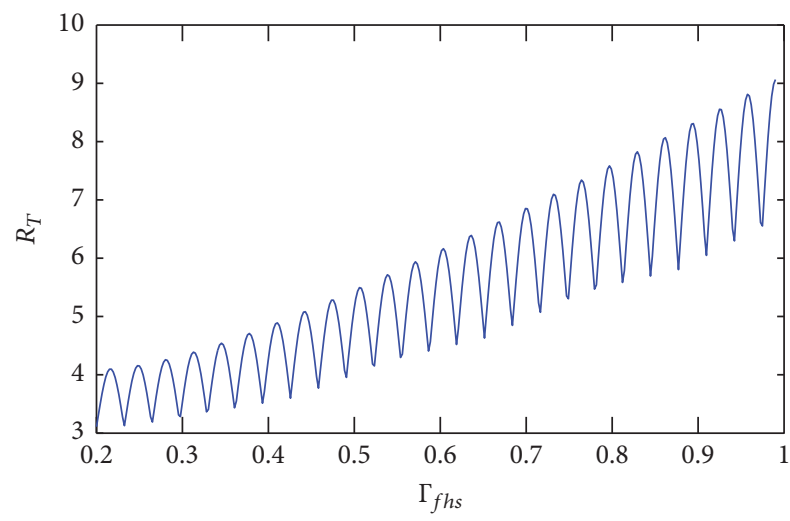

(a)

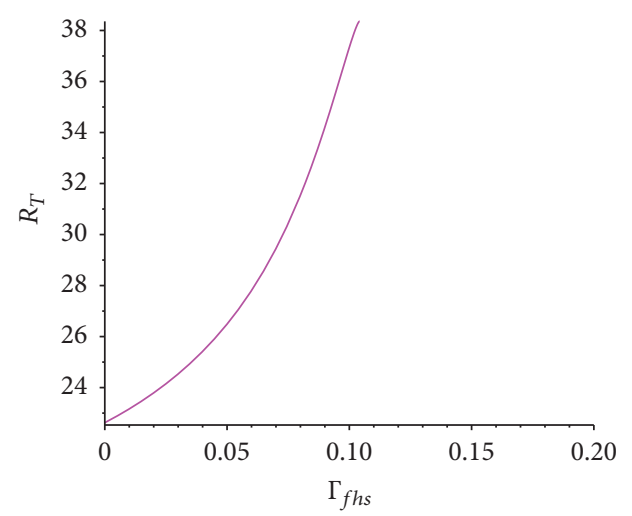

(b)

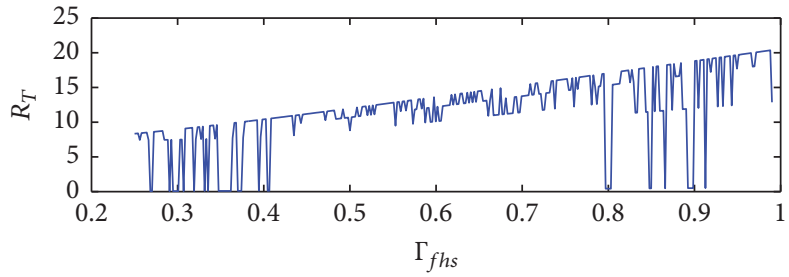

(c)

FIGURE 4: The effect of infection from $I_{F}$ to human beings with septicemic plague on the periodic reproduction number.

the parameters that are affected by seasonal weather variation are evaluated using the temperature data in Tanga (Figure 7(c)) and using the time-averaged seasonal parameters (Figure 7(b)). This result is in light of the fact that the reduction of flea population will reduce the number of individuals with bubonic and septicemic plague and as a result reduce the number of pneumonic plague infective agents that result from the progression of individual with bubonic and septicemic plague.

The reduction of flea population will reduce not only the infection from flea to other individuals but also the rate at which flea gets the disease from other individuals (human 


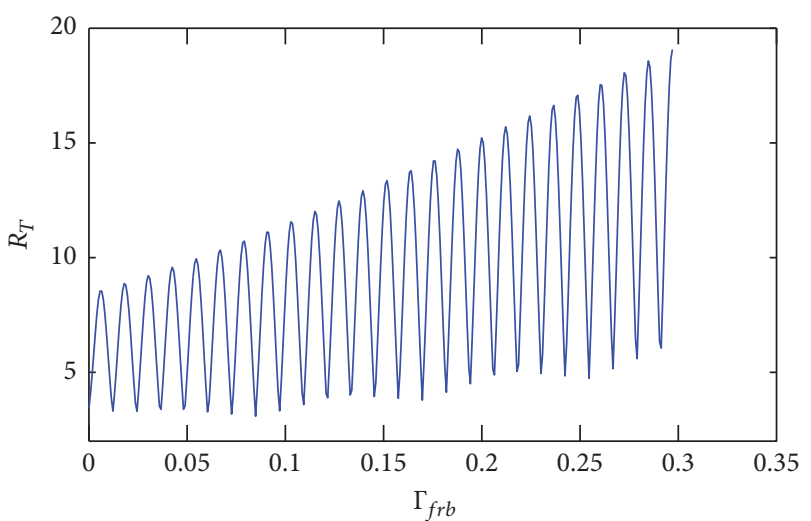

(a)

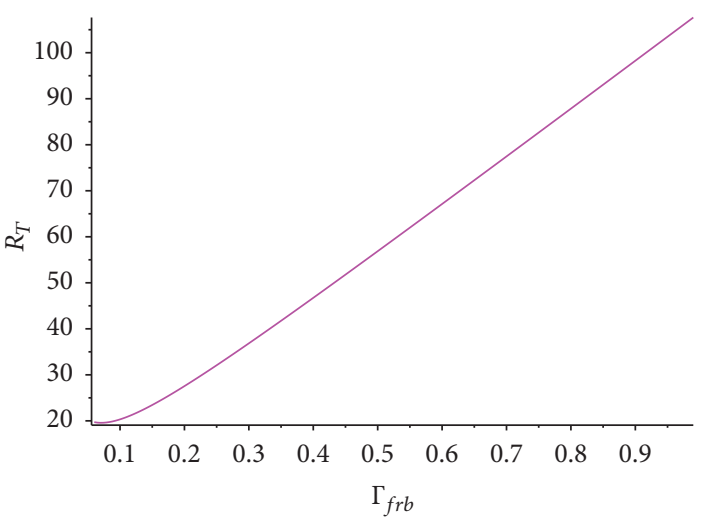

(b)

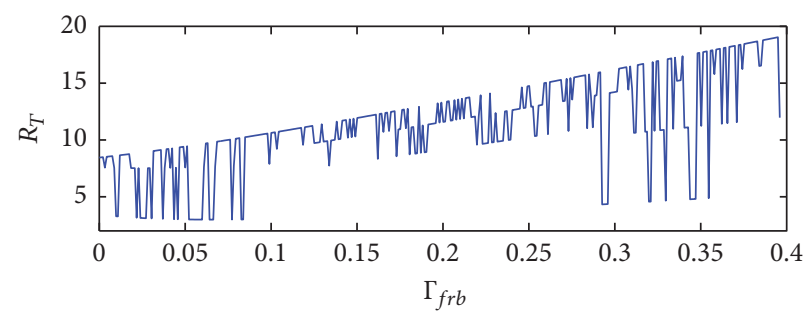

(c)

FIGURE 5: The effect of infection from $I_{F}$ to rodents with bubonic plague on the periodic reproduction number.

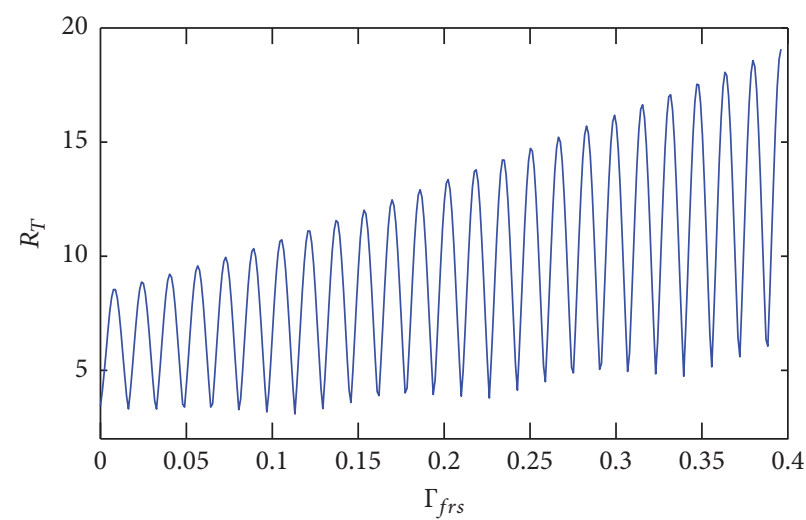

(a)

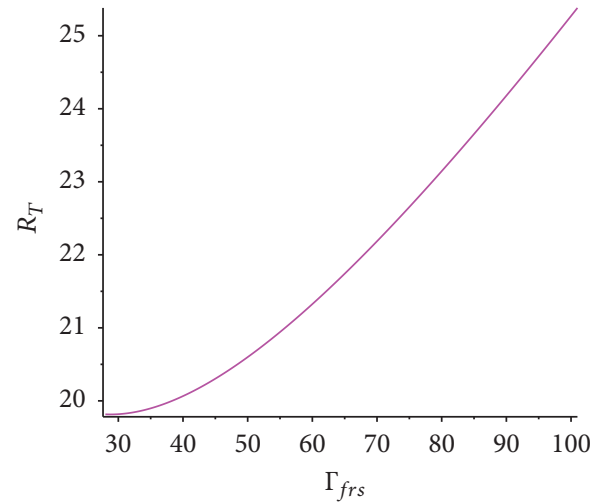

(b)

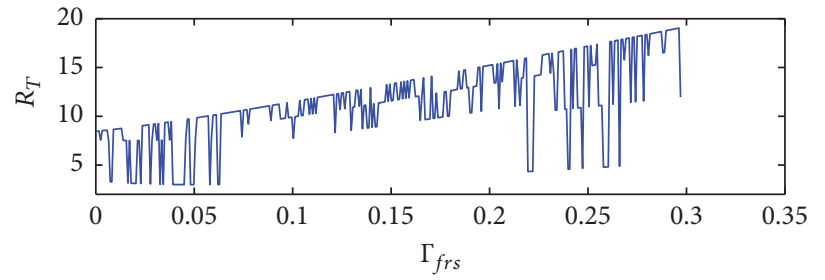

(c)

FIGURE 6: The effect of infection from $I_{F}$ to rodents with septicemic plague on the periodic reproduction number.

beings and rodents). When the flea population is reduced it will as a result reduce the interaction between susceptible fleas and other infectious individuals and vice versa. The number of fleas getting the disease increases with the increase of the rate at which fleas acquire infection from infectious human beings with bubonic plague (see Figure 8(a)) and those with septicemic plague (see Figure 9(a)). We further observe that the increase of infectious fleas may be 


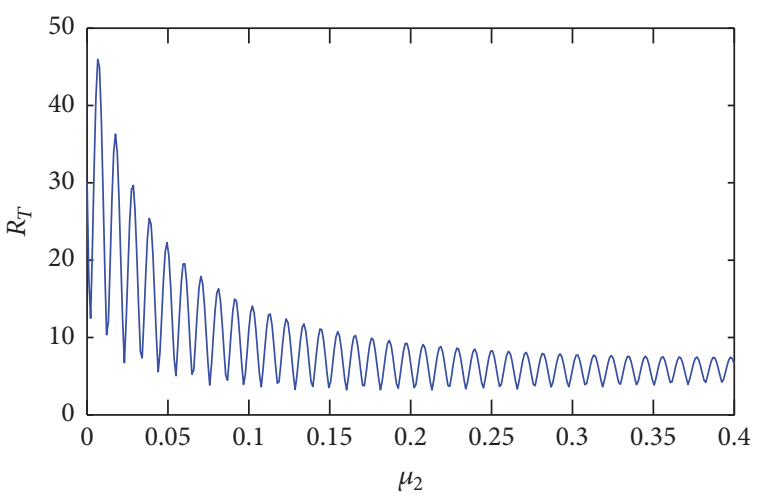

(a)

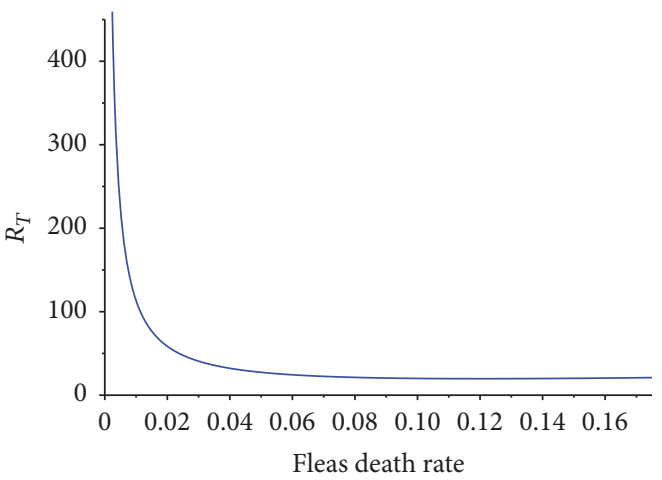

(b)

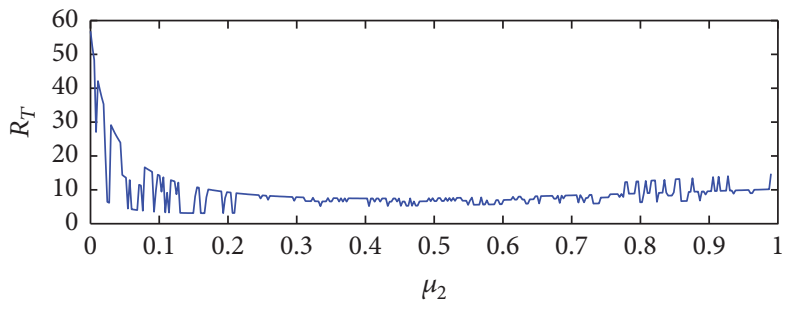

(c)

FIGURE 7: Effect of increased number of fleas' death rate on the periodic reproduction number.

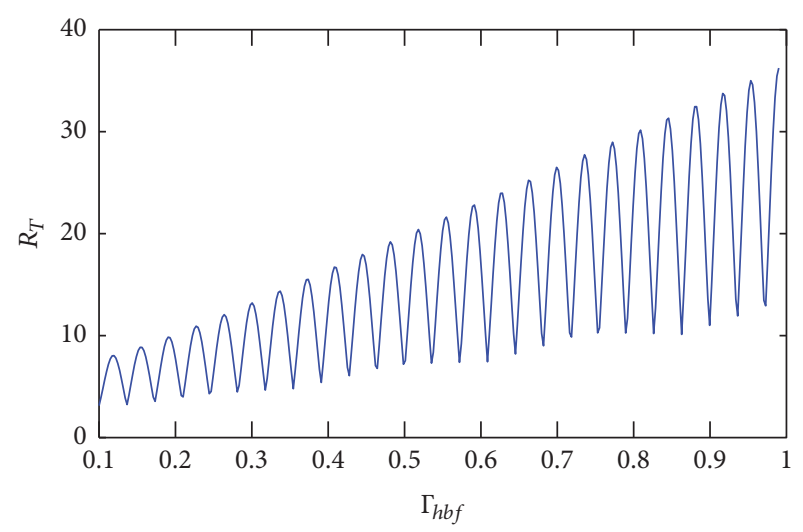

(a)

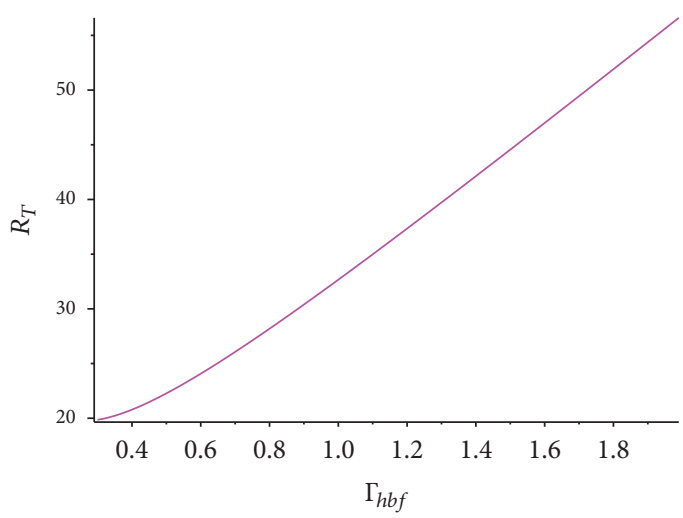

(b)

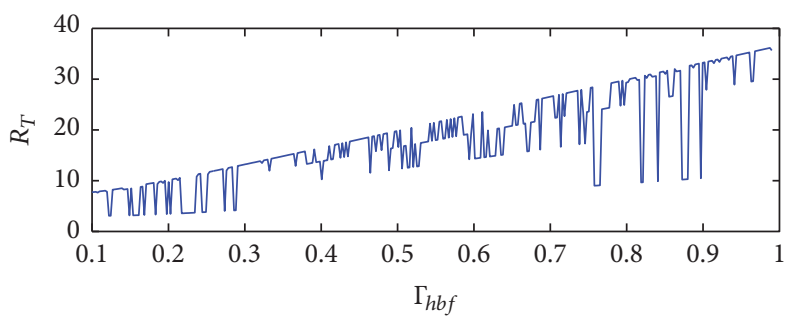

(c)

FIGURE 8: The effect of increased infection rate to fleas from the infectious human beings $\left(I_{H B}\right)$ on the periodic reproduction number.

contributed by the infectious rodents with bubonic plague (see Figure 10(a)) and those with septicemic plague (see Figure 11(a)). We can also observe the similar results when the parameters are evaluated based on the temperature data in Tanga region and when the parameters are timely averaged as in Figures 8(c), 8(b), 10(c), and 10(b) for human beings and rodents, respectively. Therefore, using these results, we settle to the point that increasing transmission rate in flea 


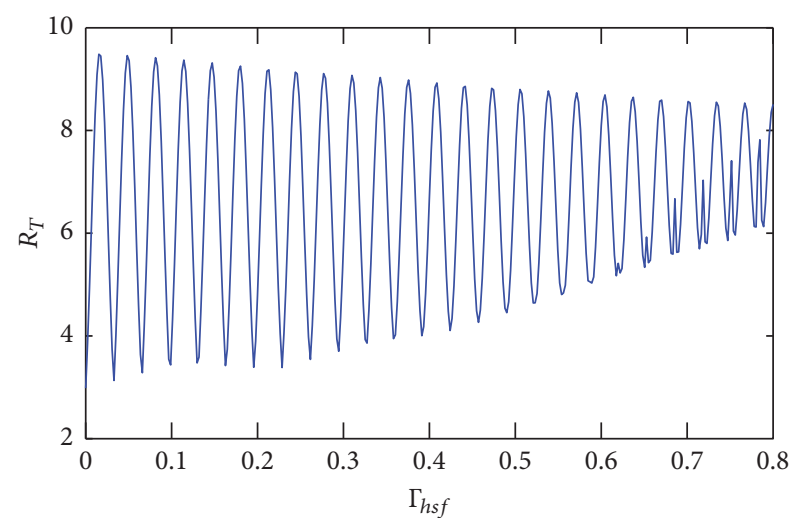

(a)

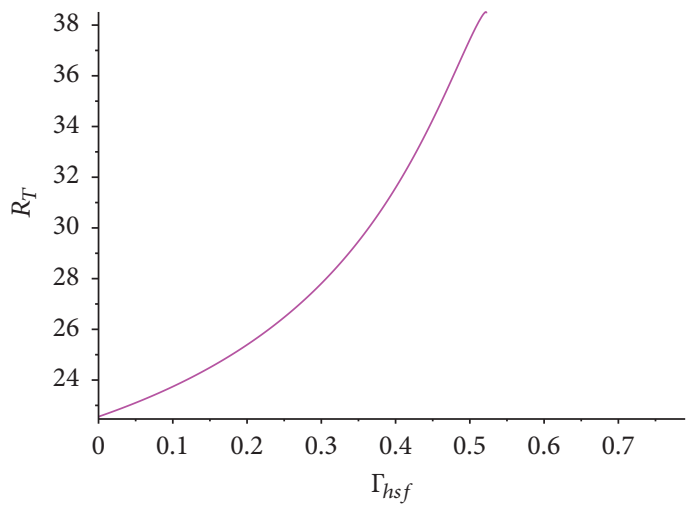

(b)

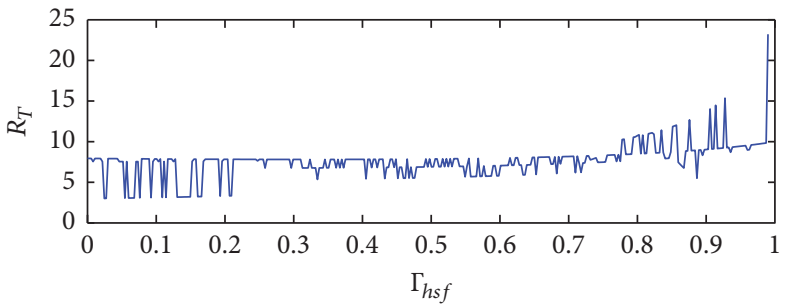

(c)

FIGURE 9: The effect of increased infection rate to fleas from the infectious human beings $\left(I_{H S}\right)$ on the periodic reproduction number.

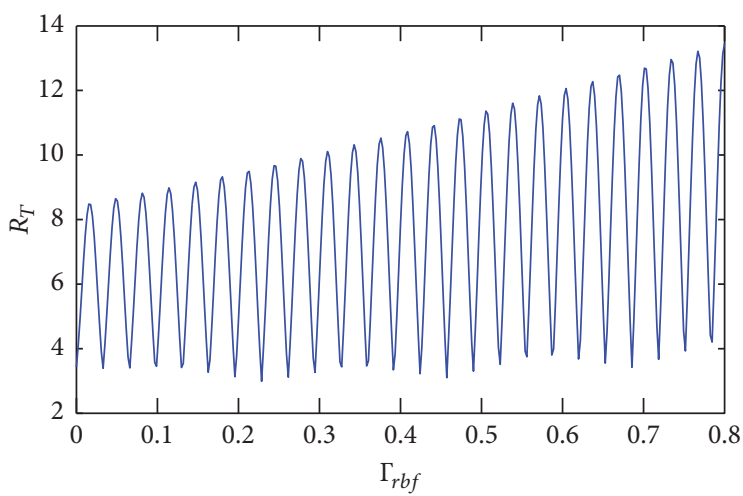

(a)

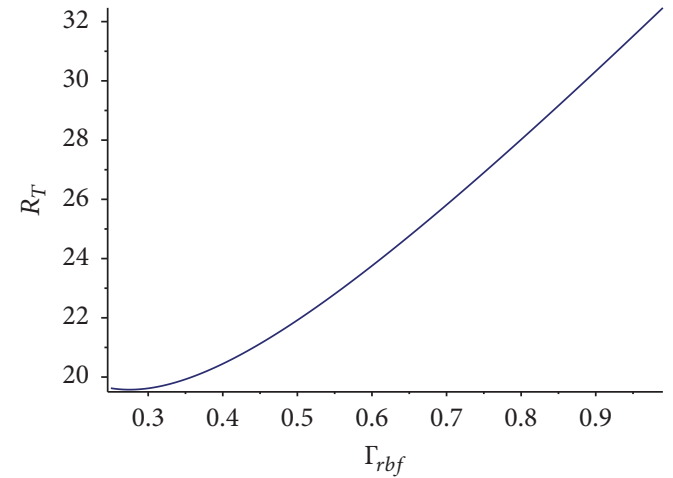

(b)

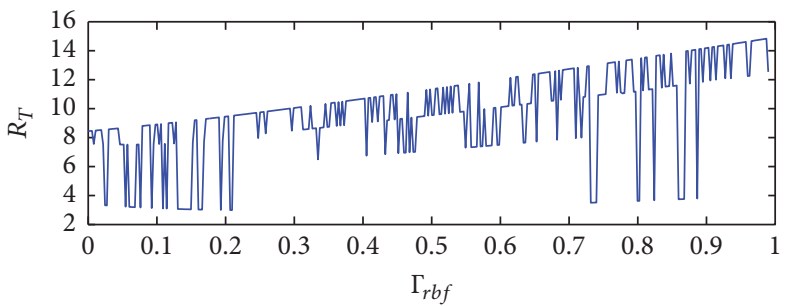

(c)

FIGURE 10: The effect of increased infection rate to fleas from the infectious rodents $\left(I_{R B}\right)$ on the periodic reproduction number.

population from human beings and rodents with bubonic and septicemic plague raises the average number of secondary plague disease infections.

Physical contact that includes sexual contact between two infectious individuals (human beings and rodents) may lead to septicemic plague. The increase in the number of individuals with septicemic plague affects the general dynamics of plague disease, particularly the average number of secondary infections. It is illustrated that increasing infection rate from a human being with septicemic plague to the other susceptible 


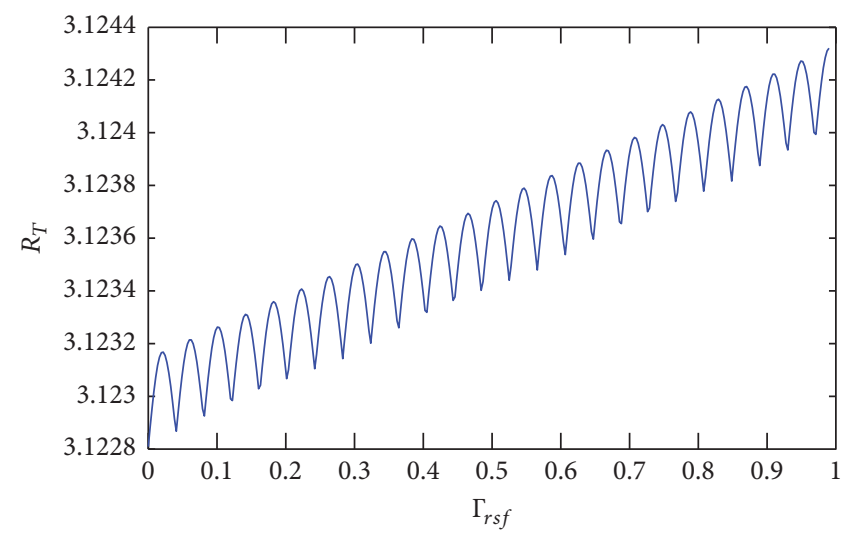

(a)

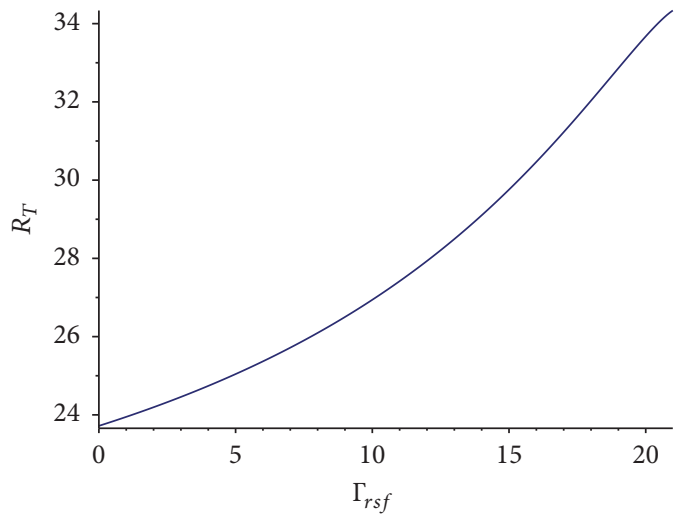

(b)

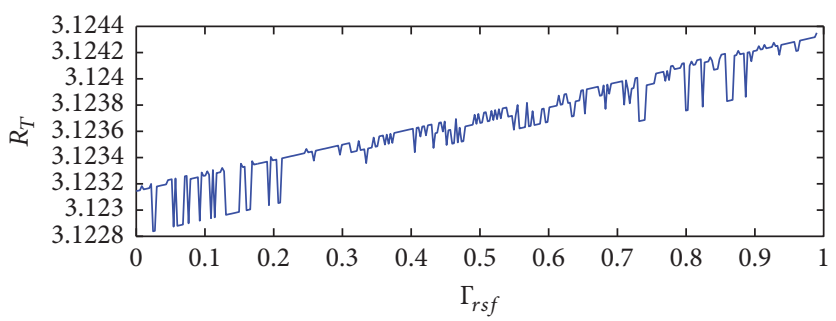

(c)

FIGURE 11: The effect of increased infection rate to fleas from the infectious rodents $\left(I_{R S}\right)$ on the periodic reproduction number.

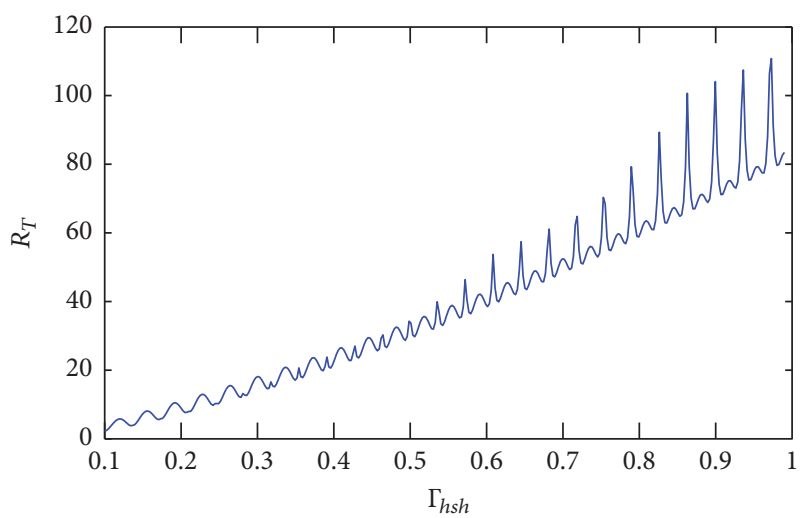

(a)

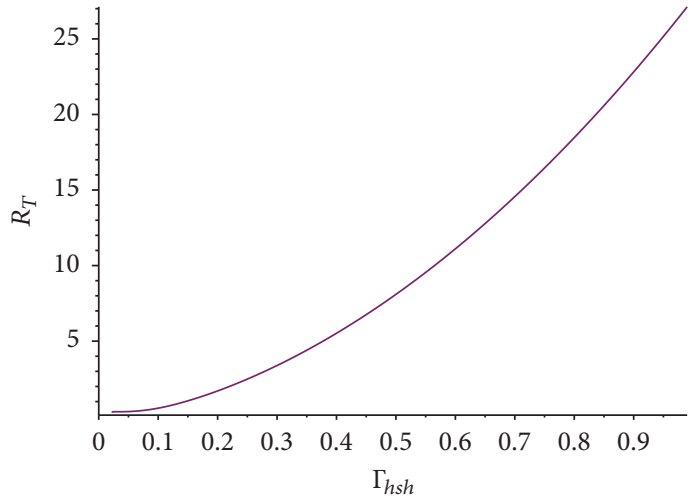

(b)

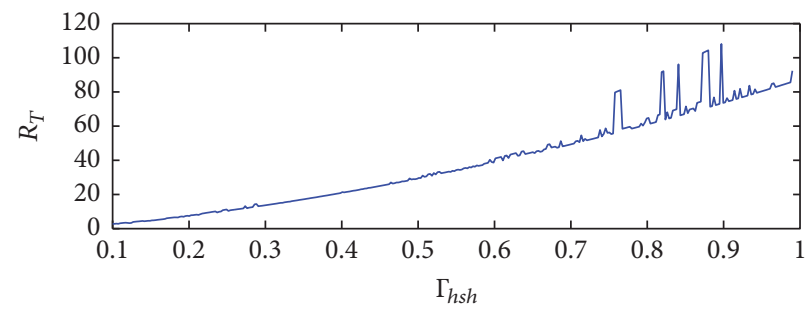

(c)

FIGURE 12: Effect of infection rate $\left(\Gamma_{h s h}\right)$ on the periodic reproduction number.

human (see Figure 12(a)) and from rodent with septicemic plague to the susceptible rodents (see Figure 13(a)) increases the average number of secondary infections. The result again shows a clear correlation when parameters are evaluated based on the temperature data from Tanga region (see Figures $12(\mathrm{c})$ and $13(\mathrm{c})$ ) and when they are averaged (see Figures 12(b) and 13(b)) for human beings and rodents, respectively. This shows the necessity to educate human beings to practice safe 


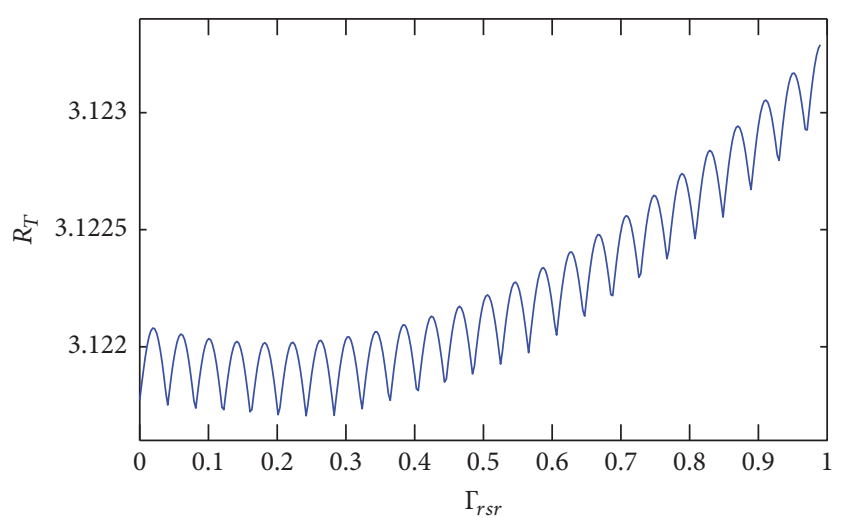

(a)

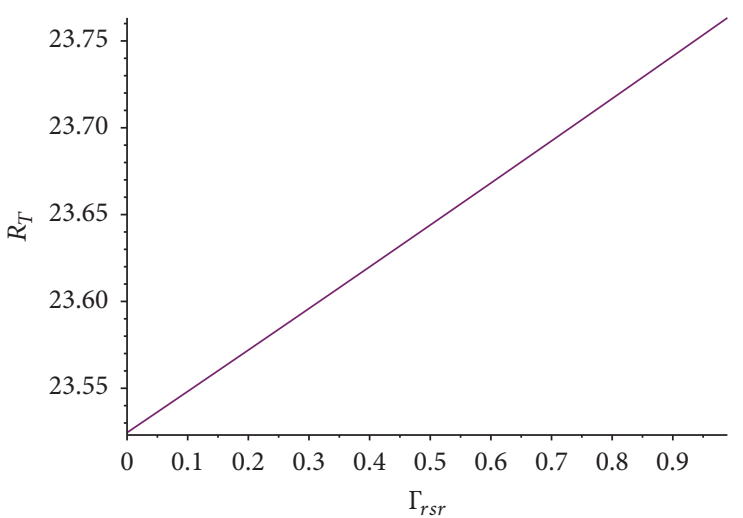

(b)

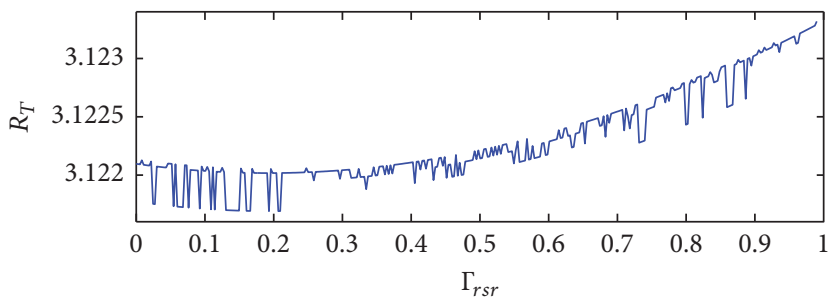

(c)

FIGURE 13: Effect of infection rate $\left(\Gamma_{r s r}\right)$ on the periodic reproduction number.

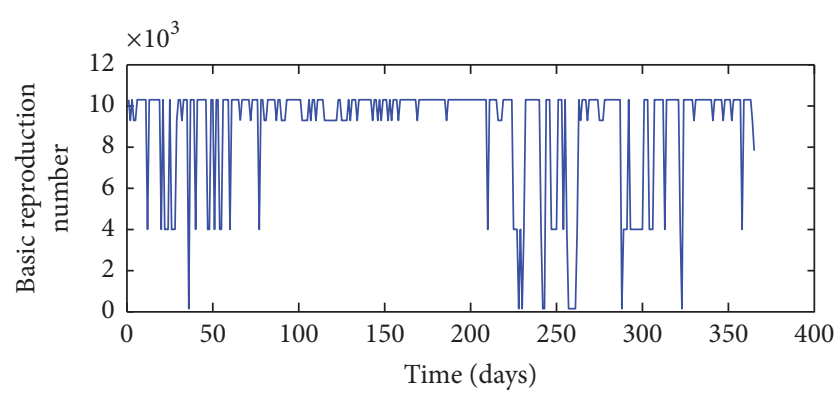

(a)

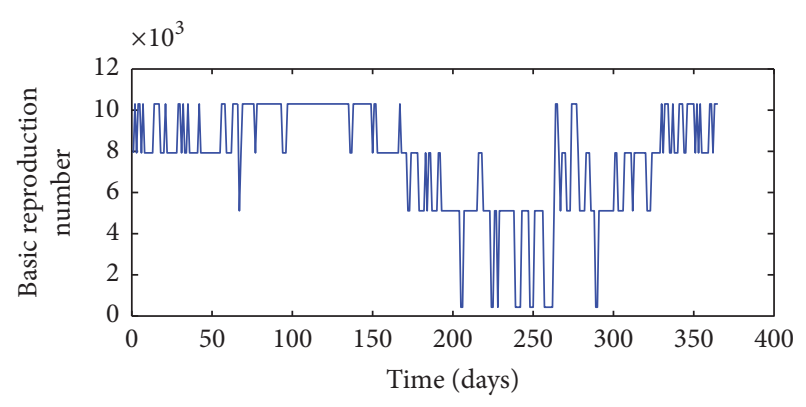

(b)

FIGURE 14: Distribution of $R_{0}$ based on fluctuation of temperature (a) and relative humidity (b) data in Kigoma.

sex using protective gears and taking necessary precaution when handling people or animals with septicemic plague. This also tells us that there is a necessity to quarantine people and animals that immigrate from areas that are infected by septicemic plague so that they do not affect other human beings or animals and thus increasing the endemicity of the disease.

The distribution of the basic reproduction number is based on the seasonal weather condition of a particular area at a particular time. This is what causes the unpredictability of the number of secondary cases of plague disease infection (bubonic, septicemic, and pneumonic plague) as it will change whenever the weather conditions change. We evaluate the distribution of the basic reproduction number based on the data we obtained on daily temperature $\left({ }^{\circ} \mathrm{C}\right)$ and humidity (\%) from five regions in Tanzania from January to December 2013. Figures 14, 15, 16, 17, and 18 show the seasonal distribution of basic reproduction number when evaluated based on the temperature and humidity data from Kigoma, Mbeya, Mtwara, Singida, and Tanga regions, respectively.

The features displayed in these results clearly show how seasonal weather fluctuation can have significant effects on the number of secondary cases of plague disease. It can be noted that there is a seasonal pattern in new plague disease infection cases. We therefore vindicate the fact that the number of plague disease infective agents peaks whenever the weather condition is favorable for plague disease transmission and it drops when the weather condition does not favor plague disease transmission.

\section{Conclusion}

The transmission of plague disease occurs in several pathways which makes the modeling of the disease challenging and 


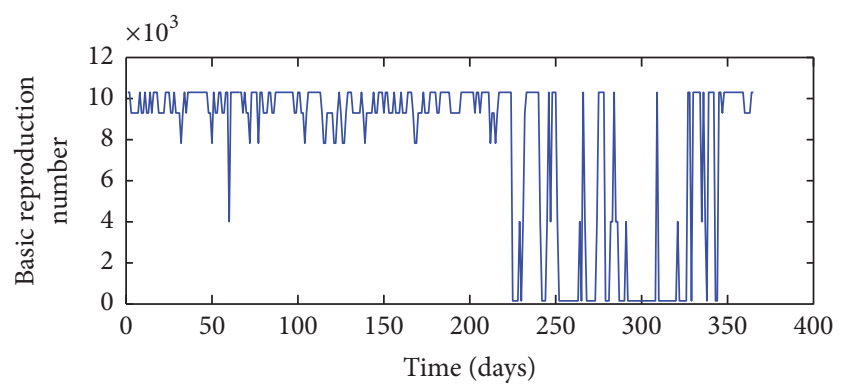

(a)

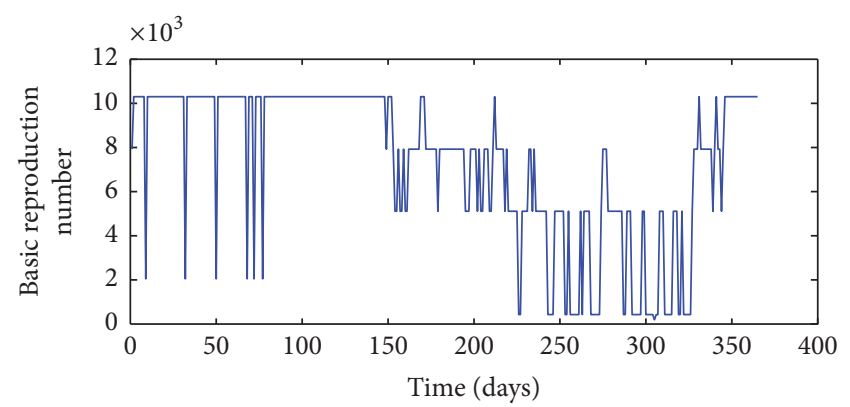

(b)

FIGURE 15: Distribution of $R_{0}$ based on fluctuation of temperature (a) and relative humidity (b) data in Mbeya.

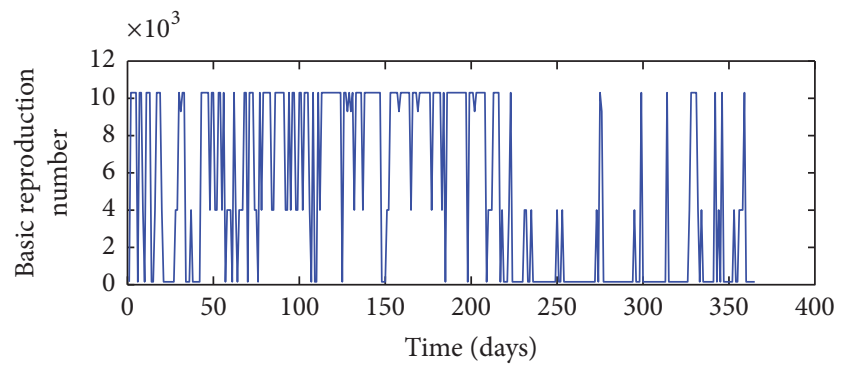

(a)

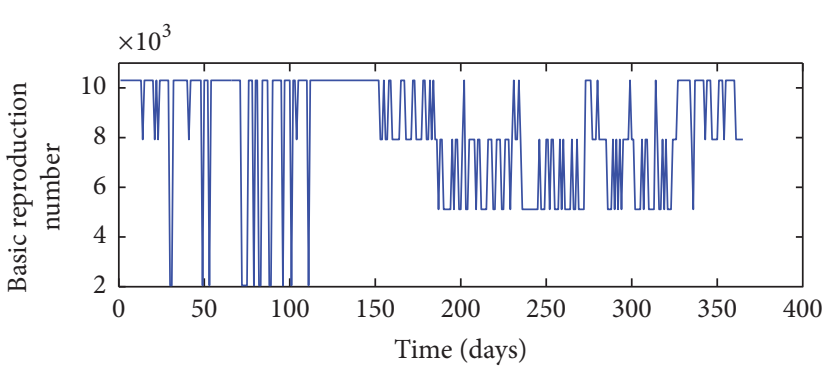

(b)

FIGURE 16: Distribution of $R_{0}$ based on fluctuation of temperature (a) and relative humidity (b) data in Mtwara.

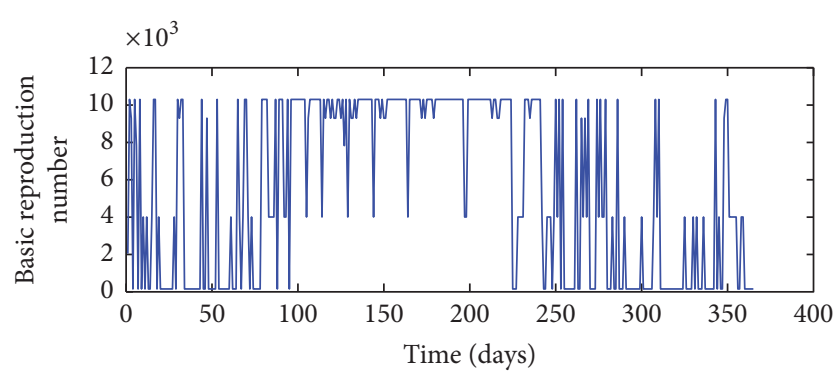

(a)

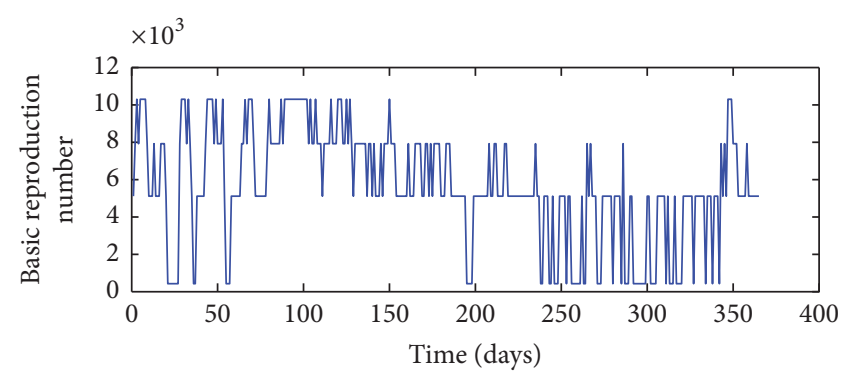

(b)

FIGURE 17: Distribution of $R_{0}$ based on fluctuation of temperature (a) and relative humidity (b) data in Singida.

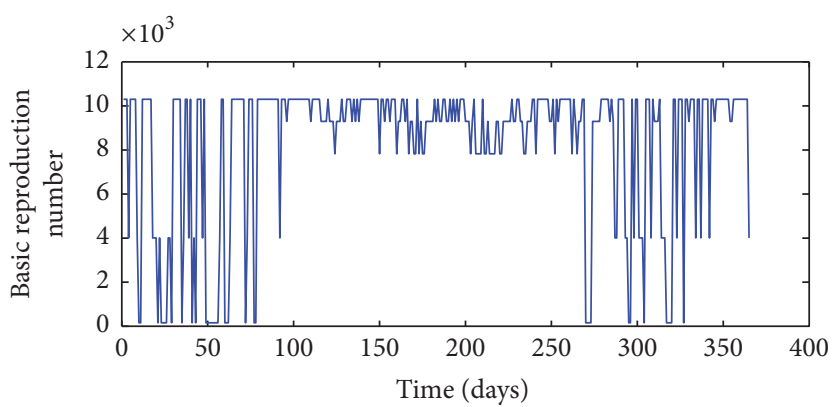

(a)

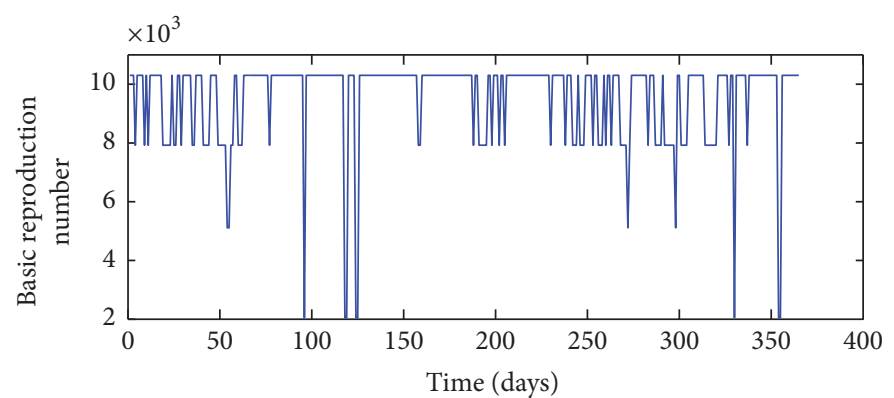

(b)

FIGURE 18: Distribution of $R_{0}$ based on fluctuation of temperature (a) and relative humidity (b) data in Tanga. 
very complex. Moreover all ways that lead to plague disease transmission are directly or indirectly affected by seasonal weather variation which causes seasonality in plague disease epidemic. The effect of seasonal weather variation has been a glowing concern in different epidemiological studies. This in turn dictates that in order to study the dynamics and propose the effective control strategies of the plague disease we must incorporate the effect of seasonal weather variation. In this study we have analysed the plague disease model incorporated with the factors that are affected by the seasonal weather variation in order to study its effects on the dynamics of the plagues disease. We have computed basic reproduction number and depicted how it can be affected by seasonal weather variation through numerical simulation. We were able to deduce that progression rates from one primary form to secondary form of plague infection, flea's infection rate, and the vector flea abundance pose the significant effect on the increase of the average number of secondary cases of plague infection. Therefore the effective control strategies must take into account these factors as they have been shown to have a significant contribution to the increase of the average number of secondary cases of plague infection.

\section{Notations}

$S_{H}$ : Susceptible human population

$E_{H}$ : Exposed human population

$I_{H B}$ : Infectious human population with bubonic plague

$I_{H S}$ : Infectious human population with septicemic plague

$I_{H P}$ : Infectious human population with pneumonic plague

$R_{H}$ : Recovered human population

$S_{R}$ : Susceptible rodents

$E_{R}$ : Exposed rodents

$I_{R B}$ : Infectious rodents with bubonic plague

$I_{R S}:$ Infectious rodents with septicemic plague

$I_{R P}$ : Infectious rodents with pneumonic plague

$S_{F}$ : Susceptible fleas

$I_{F}:$ Infected fleas

A: Pathogens in the soil/environment.

\section{Conflicts of Interest}

The authors declare that there are no conflicts of interest regarding the publication of this paper.

\section{References}

[1] D. M. Wagner, J. Klunk, M. Harbeck et al., "Yersinia pestis and the Plague of Justinian 541-543 AD: A genomic analysis," The Lancet Infectious Diseases, vol. 14, no. 4, pp. 319-326, 2014.

[2] S. Altizer, A. Dobson, P. Hosseini, P. Hudson, M. Pascual, and P. Rohani, "Seasonality and the dynamics of infectious diseases," Ecology Letters, vol. 9, no. 4, pp. 467-484, 2006.

[3] D. C. Cavanaugh and J. D. Marshall Jr., "The influence of climate on the seasonal prevalence of plague in the republic of vietnam," Journal of Wildlife Diseases, vol. 8, no. 1, pp. 85-94, 1972.

[4] J. Silverman, M. K. Rust, and D. A. Reierson, "Influence of temperature and humidity on survival and development of the cat flea, Ctenocephalides felis (Siphonaptera: Pulicidae)., Journal of Medical Entomology, vol. 18, no. 1, pp. 78-83, 1981.

[5] D. Zentko and D. Richman, Cat Flea, Ctenocephalides Felis Felis (Bouché), Citeseer, 1997.

[6] D. C. Cavanaugh, "Specific effect of temperature upon transmission of the plague bacillus by the oriental rat flea, xenopsylla cheopis," The American Journal of Tropical Medicine and Hygiene, vol. 20, no. 2, pp. 264-273, 1971.

[7] A. W. Bacot and C. J. Martin, "The respective influences of temperature and moisture upon the survival of the rat flea (xenopsylla cheopis) away from its host," Journal of Hygiene, vol. 23, no. 1, pp. 98-105, 1924.

[8] K. L. Gage, T. R. Burkot, R. J. Eisen, and E. B. Hayes, "Climate and vectorborne diseases," American Journal of Preventive Medicine, vol. 35, no. 5, pp. 436-450, 2008.

[9] R. E. Enscore, B. J. Biggerstaff, T. L. Brown et al., "Modeling relationships between climate and the frequency of human plague cases in the southwestern united states, 1960-1997," The American Journal of Tropical Medicine and Hygiene, vol. 66, no. 2, pp. 186-196, 2002.

[10] R. S. J. Brooks, "The influence of saturation deficiency and of temperature on the course of epidemic plague," The Journal of hygiene, supplement 15, pp. 881-889, 1917.

[11] R. J. Eisen and K. L. Gage, "Adaptive strategies of yersinia pestis to persist during inter-epizootic and epizootic periods," Veterinary Research, vol. 40, no. 2, article no. 01, 2009.

[12] B. R. Krasnov, I. S. Khokhlova, L. J. Fielden, and N. V. Burdelova, "Effect of Air Temperature and Humidity on the Survival of PreImaginal Stages of Two Flea Species (Siphonaptera: Pulicidae)," Journal of Medical Entomology, vol. 38, no. 5, pp. 629-637, 2001.

[13] R. R. Parmenter, E. P. Yadav, C. A. Parmenter, P. Ettestad, and K. L. Gage, "Incidence of plague associated with increased winterspring precipitation in new mexico," The American Journal of Tropical Medicine and Hygiene, vol. 61, no. 5, pp. 814-821, 1999.

[14] L. Korslund and H. Steen, "Small rodent winter survival: Snow conditions limit access to food resources," Journal of Animal Ecology, vol. 75, no. 1, pp. 156-166, 2006.

[15] S. Roberts, J. Van Wagtendonk, A. Miles, D. Kelt, and J. Lutz, "Modeling the effects of fire severity and spatial complexity on small mammals in yosemite national park, california," Fire Ecology Special Issue, vol. 4, pp. 84-104, 2008.

[16] F. M. Jaksic and M. Lima, "Myths and facts on ratadas: Bamboo blooms, rainfall peaks and rodent outbreaks in South America," Austral Ecology, vol. 28, no. 3, pp. 237-251, 2003.

[17] P. L. Meserve, W. Bryan Milstead, and J. R. Gutiérrez, "Results of a food addition experiment in a north-central Chile small mammal assemblage: Evidence for the role of "bottom-up" factors," Oikos, vol. 94, no. 3, pp. 548-556, 2001.

[18] M. Letnic, B. Tamayo, and C. R. Dickman, "The responses of mammals to La Niña (El Niño Southern Oscillation)-associated rainfall, predation, and wildfire in central Australia," Journal of Mammalogy, vol. 86, no. 4, pp. 689-703, 2005.

[19] H. Leirs, R. Verhagen, W. Verheyen, P. Mwanjabe, and T. Mbise, "Forecasting rodent outbreaks in africa: an ecological basis for mastomys control in tanzania," Journal of Applied Ecology, pp. 937-943, 1996.

[20] C. R. Dickman, P. S. Mahon, P. Masters, and D. F. Gibson, "Longterm dynamics of rodent populations in arid Australia: The influence of rainfall," Wildlife Research, vol. 26, no. 4, pp. 389403, 1999. 
[21] S. L. Agar, J. Sha, S. M. Foltz et al., "Characterization of the rat pneumonic plague model: infection kinetics following aerosolization of Yersinia pestis CO92," Microbes and Infection, vol. 11, no. 2, pp. 205-214, 2009.

[22] B. R. Krasnov, I. S. Khokhlova, L. J. Fielden, and N. V. Burdelova, "Development rates of two Xenopsylla flea species in relation to air temperature and humidity," Medical and Veterinary Entomology, vol. 15, no. 3, pp. 249-258, 2001.

[23] B. R. Krasnov, N. V. Burdelova, G. I. Shenbrot, and I. S. Khokhlova, "Annual cycles of four flea species in the central Negev desert," Medical and Veterinary Entomology, vol. 16, no. 3, pp. 266-276, 2002.

[24] D. Davis, "Plague in Africa from 1935 to 1949," WHO Bulletin, vol. 9, no. 5, pp. 665-669, 1953.

[25] E. Mitscherlich and E. H. Marth, Microbial Survival in The Environment: Bacteria and Rickettsiae Important in Human and Animal Health, Springer Science \& Business Media, 2012.

[26] S. Ayyadurai, L. Houhamdi, H. Lepidi et al., "Long-term persistence of virulent Yersinia pestis in soil," Microbiology, vol. 154, no. 9, pp. 2865-2871, 2008.

[27] H. Mollaret, "Experimental Conservation of Plague in The Soil," DTIC Document, 1964.

[28] R. J. Eisen, J. M. Petersen, C. L. Higgins et al., "Persistence of Yersinia pestis in soil under natural conditions," Emerging Infectious Diseases, vol. 14, no. 6, pp. 941-943, 2008.

[29] M. Nozadze, E. Zhgenti, M. Meparishvili et al. et al., "Comparative proteomic studies of yersinia pestis strains isolated from natural foci in the republic of georgia," Frontiers in Public Health, vol. 3, pp. 145-239, 2015.

[30] J. Koirala, "Plague: Disease, Management, and Recognition of Act of Terrorism," Infectious Disease Clinics of North America, vol. 20, no. 2, pp. 273-287, 2006.

[31] R. R. Brubaker, "The genus Yersinia: biochemistry and genetics of virulence.," Current Topics in Microbiology and Immunology, vol. 57, pp. 111-158, 1972.

[32] N. C. Stenseth, B. B. Atshabar, M. Begon et al., "Plague: Past, present, and future," PLoS Medicine, vol. 5, no. 1, pp. 0009-0013, 2008.

[33] A. Bevelacqua and R. Stilp, Terrorism Handbook for Operational Responders, Cengage Learning, 2009.

[34] K. F. Meyer, "Pneumonic plague," Bacteriological Reviews, vol. 25, article 249, no. 3, 1961.

[35] L. J. Rose, R. Donlan, S. N. Banerjee, and M. J. Arduino, "Survival of Yersinia pestis on environmental surfaces," Applied and Environmental Microbiology, vol. 69, no. 4, pp. 2166-2171, 2003.

[36] R. J. Eisen, A. P. Wilder, S. W. Bearden, J. A. Montenieri, and K. L. Gage, "Early-phase transmission of Yersinia pestis by unblocked Xenopsylla cheopis (Siphonaptera: Pulicidae) is as efficient as transmission by blocked fleas," Journal of Medical Entomology, vol. 44, no. 4, pp. 678-682, 2007.

[37] R. Gani and S. Leach, "Epidemiologic Determinants for Modeling Pneumonic Plague Outbreaks," Emerging Infectious Diseases, vol. 10, no. 4, pp. 608-614, 2004.

[38] M. J. Keeling and C. A. Gilligan, "Bubonic plague: A metapopulation model of a zoonosis," Proceedings of the Royal Society B: Biological Sciences, vol. 267, no. 1458, pp. 2219-2230, 2000.

[39] K. J. Kugeler, J. E. Staples, A. F. Hinckley, K. L. Gage, and P. S. Mead, "Epidemiology of human plague in the United States, 1900-2012," Emerging Infectious Diseases, vol. 21, no. 1, pp. 1622, 2015.
[40] C. Tollenaere, L. Rahalison, M. Ranjalahy et al., "Susceptibility to Yersinia pestis experimental infection in wild rattus rattus, reservoir of plague in Madagascar," EcoHealth, vol. 7, no. 2, pp. 242-247, 2010.

[41] S. Morand and P. H. Harvey, "Mammalian metabolism, longevity and parasite species richness," Proceedings of the Royal Society B: Biological Sciences, vol. 267, no. 1456, pp. 1999-2003, 2000.

[42] P. Hunter, "Climate change and waterborne and vector-borne disease," Journal of Applied Microbiology, vol. 94, no. s1, pp. 3746, 2003.

[43] Y. Dumont, F. Chiroleu, and C. Domerg, "On a temporal model for the Chikungunya disease: modeling, theory and numerics," Mathematical Biosciences, vol. 213, no. 1, pp. 80-91, 2008.

[44] S. C. Mpeshe, L. S. Luboobi, and Y. Nkansah-Gyekye, "Modeling the impact of climate change on the dynamics of rift valley fever," Computational and Mathematical Methods in Medicine, Article ID 627586, Art. ID 627586, 12 pages, 2014.

[45] G. Aronsson and R. B. Kellogg, "On a differential equation arising from compartmental analysis," Mathematical Biosciences. An International Journal, vol. 38, no. 1-2, pp. 113-122, 1978.

[46] W. Wang and X.-Q. Zhao, "Threshold dynamics for compartmental epidemic models in periodic environments," Journal of Dynamics and Differential Equations, vol. 20, no. 3, pp. 699-717, 2008.

[47] P. van den Driessche and J. Watmough, "Reproduction numbers and sub-threshold endemic equilibria for compartmental models of disease transmission," Mathematical Biosciences, vol. 180, pp. 29-48, 2002.

[48] J. K. Hale, Ordinary Differential Equations, John Wiley and Sons, Inc, New York, NY, USA, 1980.

[49] O. Diekmann, J. A. Heesterbeek, and J. A. Metz, "On the definition and the computation of the basic reproduction ratio $R_{0}$ in models for infectious diseases in heterogeneous populations," Journal of Mathematical Biology, vol. 28, no. 4, pp. 365-382, 1990.

[50] H. L. Smith and P. Waltman, The Theory of the Chemostat, Cambridge University Press, 1995.

[51] T. S. Shores, Applied Linear Algebra and Matrix Analysis, Springer Science \& Business Media, 2007.

[52] C. L. Wesley and L. J. Allen, "The basic reproduction number in epidemic models with periodic demographics," Journal of Biological Dynamics, vol. 3, no. 2-3, pp. 116-129, 2009.

[53] J. Heesterbeek, Mathematical Epidemiology of Infectious Diseases: Model Building, Analysis and Interpretation, vol. 5, John Wiley \& Sons, 2000.

[54] M. H. Gail and J. Benichou, Encyclopedia of Epidemiologic Methods, John Wiley \& Sons, 2000.

[55] N. A. Hartemink, S. E. Randolph, S. A. Davis, and J. A. P. Heesterbeek, "The basic reproduction number for complex disease systems: Defining R 0 for tick-borne infections," American Naturalist, vol. 171, no. 6, pp. 743-754, 2008.

[56] J. Li, D. Blakeley, and R. J. Smith, “The failure of $R_{0}$," Computational and Mathematical Methods in Medicine, vol. 2011, Article ID 527610, 17 pages, 2011.

[57] J. A. Rozendaal, Vector Control: Methods for Use by Individuals and Communities, World Health Organization, 1997. 


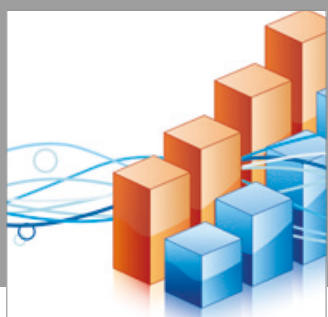

Advances in

Operations Research

vatersals

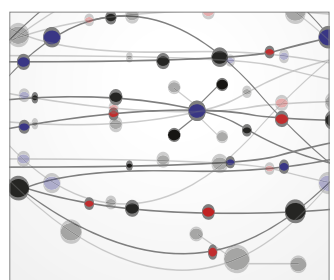

\section{The Scientific} World Journal
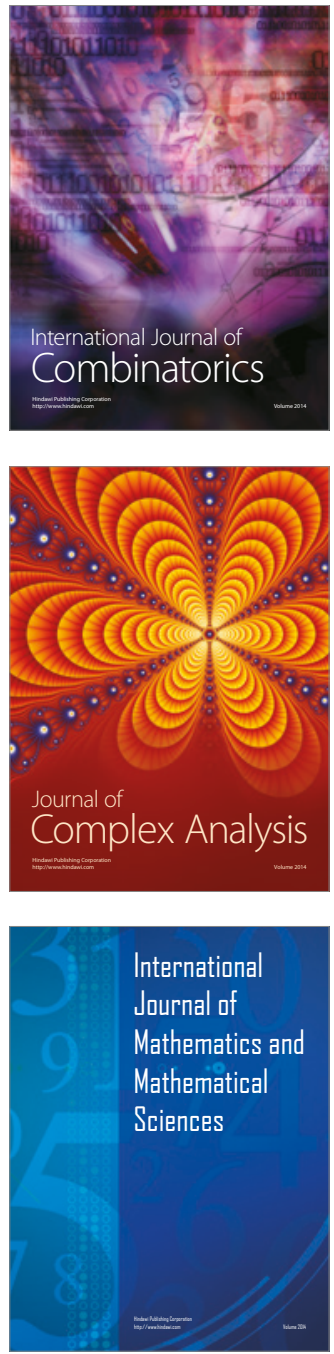
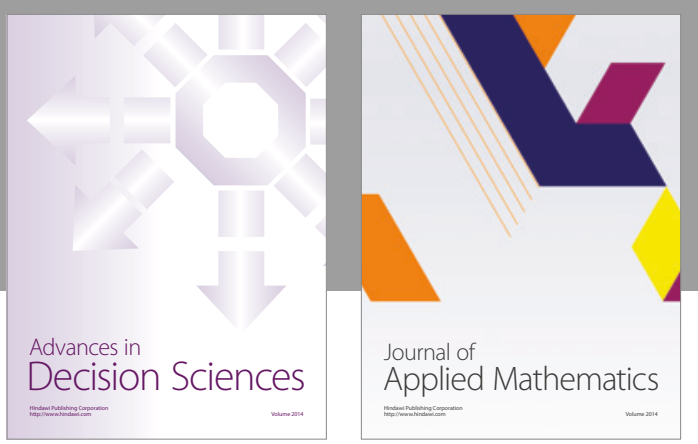

Algebra

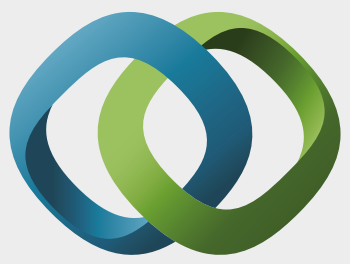

\section{Hindawi}

Submit your manuscripts at

https://www.hindawi.com
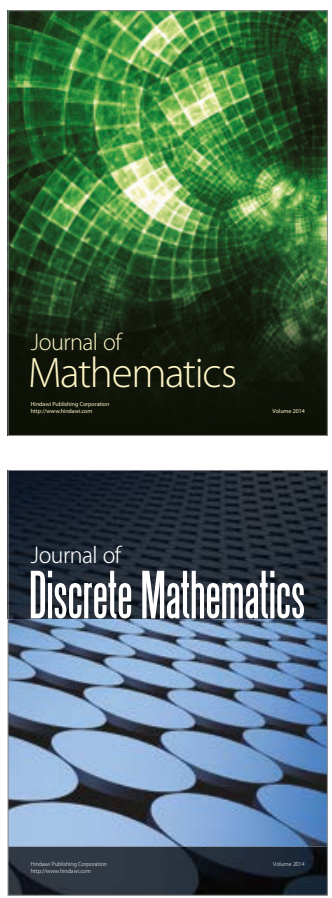

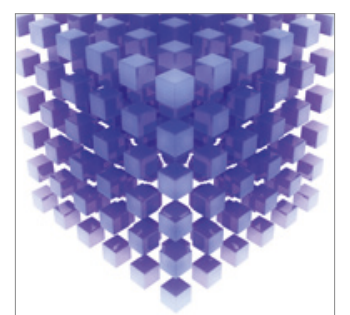

Mathematical Problems in Engineering
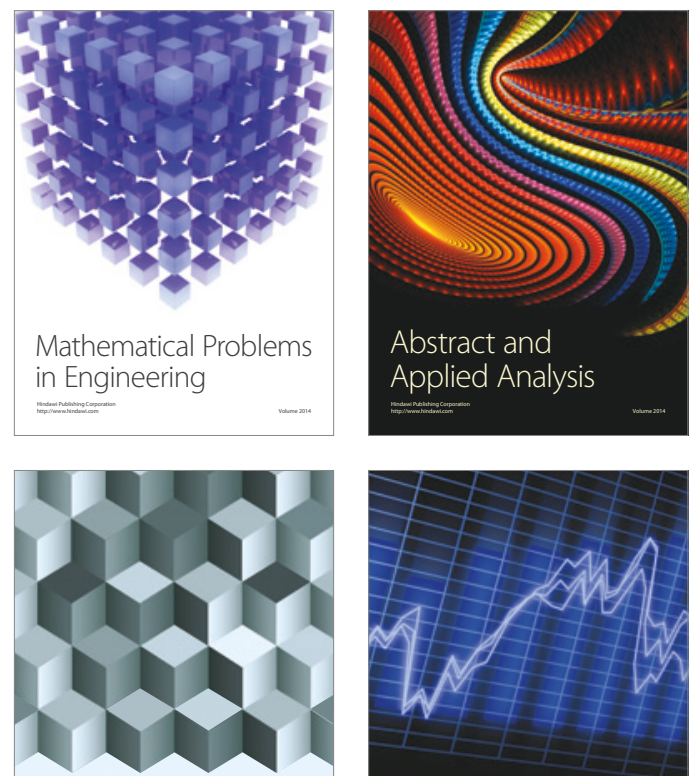

Journal of

Function Spaces

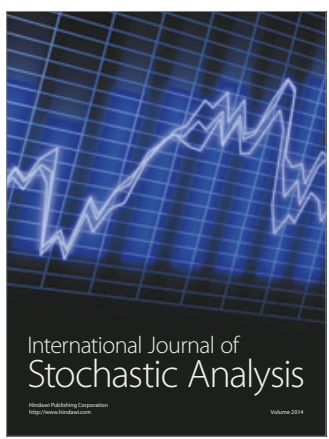

Probability and Statistics
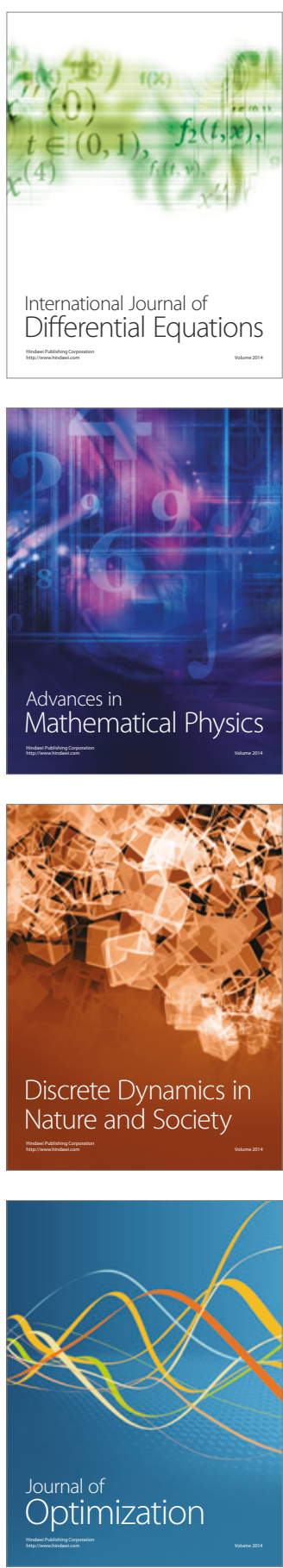